\title{
Some Families of Orthogonal Polynomials of a Discrete Variable and their Applications to Graphs and Codes*
}

\author{
M. Cámara, J. Fàbrega, M.A. Fiol and E. Garriga \\ Departament de Matemàtica Aplicada IV \\ Universitat Politècnica de Catalunya \\ Jordi Girona 1-3, Mòdul C3, Campus Nord \\ 08034 Barcelona, Catalonia (Spain).
}

Submitted: Sep 15, 2008; Accepted: Jun 25, 2009; Published: Jul 9, 2009

Mathematics Subject Classification: 05C50, 05E30, 05E35

\begin{abstract}
We present some related families of orthogonal polynomials of a discrete variable and survey some of their applications in the study of (distance-regular) graphs and (completely regular) codes. One of the main peculiarities of such orthogonal systems is their non-standard normalization condition, requiring that the square norm of each polynomial must equal its value at a given point of the mesh. For instance, when they are defined from the spectrum of a graph, one of these families is the system of the predistance polynomials which, in the case of distance-regular graphs, turns out to be the sequence of distance polynomials. The applications range from (quasi-spectral) characterizations of distance-regular graphs, walk-regular graphs, local distance-regularity and completely regular codes, to some results on representation theory.
\end{abstract}

\section{Introduction}

The theory of orthogonal polynomials is a well-developed area of mathematics and it has a large number of applications in the study of both theoretical and practical problems.

\footnotetext{
*Research supported by the "Ministerio de Ciencia e Innovación" (Spain) with the European Regional Development Fund under project MTM2008-06620-C03-01/MTM and by the Catalan Research Council under project 2005SGR00256.

Part of this work was presented in the Int. Conf. Alhambra'2000 (Invited Conf. in the 'Symposium on Orthogonal Polynomials'), Granada, Spain, July 3-7, 2000.

Emails: mcamara@ma4.upc.edu, jfabrega@ma4.upc.edu, fiol@mat.upc.es, egarriga@mat.upc.es
} 
Two standard references on this topic are the classic textbook of Szegö [27] and Chihara's work [4] which puts more emphasis on the discrete case.

In particular, different sequences of orthogonal polynomials have important applications in algebraic combinatorics. For instance, as described by Godsil [24], some examples of such sequences are the matching polynomials of the complete graphs and the rook polynomials of the complete bipartite graphs. Another example studied in the same reference is the application of orthogonal polynomials in the study of polynomial spaces.

In the area of graph theory, we find the Chebyshev polynomials (of the second kind) when studying the characteristic polynomials of the paths. Another recent application in this area has been given by Chung, Faber and Manteuffel [5], and Van Dam and Haemers [9], who gave upper bounds on the diameter of a graph in terms of its spectrum by using Chebyshev polynomials shifted to a proper interval.

In the same area, one of the most important applications of orthogonal polynomials, not always sufficiently exploited in opinion of the authors, is the study of distanceregularity of graphs and related codes. Since their introduction, distance-regular graphs and their main generalization, the association schemes, have proved to be a key concept in algebraic combinatorics. They have important connections with other branches of mathematics, such as geometry, coding theory, group theory, design theory, as well as with other areas of graph theory. As stated in the preface of the comprehensive textbook of Brouwer, Cohen and Neumaier [3], this is because most finite objects bearing "enough regularity" are closely related to certain distance-regular graphs. Other basic references on distanceregular graphs are Biggs [2], with a good introduction to distance-transitive graphs, and Bannai and Ito [1] where the connection with the theory of association schemes is emphasized.

The aim of this paper is to convince the reader of the importance and possibilities of some families of orthogonal polynomials in the study of different (local and global) concepts of distance-regularity in graphs. With this aim, we describe in detail three such families and survey some of their recent applications. We believe that these applications do not exhaust all the possibilities and we hope that many more will be given in the near future. One of the more recent application of one of these families, the so-called predistance polynomials, has arisen in the study of a kind of partial distance-regularity in graphs (the so-called $k$-walk-regular graphs), which include as extreme cases both distance-regular and walk-regular graphs; see [6].

The paper is organized as follows. In the next section we present some new families of orthogonal polynomials of a discrete variable, which have recently proven very useful in the study of distance-regular graphs and their related concepts, such as local distanceregularity and completely regular codes. One of the main peculiarities of such orthogonal systems is their non-standard normalization condition, requiring that the square norm of each polynomial must equal its value at a given point of the mesh. Then, to illustrate the usefulness of such polynomials, we discuss in Section 3 some of their applications in the area of algebraic graph theory. More specifically, we study some results concerning the concept of distance-regularity, defined in Subsection 3.3, and we will survey some results obtained from the study of two different meshes and their corresponding orthogonal 
canonical systems: the local spectrum of a given graph, defined in Subsection 3.2, and the spectrum of a subset of its vertex set, defined in Subsection 3.7.

\section{On orthogonal polynomials of a discrete variable}

In this section we begin by surveying some known results about orthogonal polynomials of a discrete variable. In order to make this paper more accessible to readers not familiar with this topic, we have included all the proofs. Afterwards, we describe three new families of such polynomials: the canonical orthogonal system, their dual polynomials and their conjugates. We begin by presenting some notation and basic facts.

Let $\mathcal{M}:=\left\{\lambda_{0}, \lambda_{1}, \cdots, \lambda_{d}\right\}, \lambda_{0}>\lambda_{1}>\cdots>\lambda_{d}$, be a mesh of real numbers. A real function of a discrete variable $f: \mathcal{M} \longrightarrow \mathbb{R}$ can be seen as the restriction on $\mathcal{M}$ of a number of functions of real variable. Moreover, if we only consider polynomial functions, the class of possible extensions of one discrete function on $\mathcal{M}$ constitute an element of the quotient algebra $\mathbb{R}[x] /(Z)$ where $(Z)$ is the ideal generated by the polynomial $Z:=\prod_{l=0}^{d}\left(x-\lambda_{l}\right)$. Each class has a unique canonical representative of degree at most $d$. Denoting by $\mathcal{F}(\mathcal{M})$ the set of functions on the mesh, we then have the following natural identifications:

$$
\mathcal{F}(\mathcal{M}) \longleftrightarrow \mathbb{R}[x] /(Z) \longleftrightarrow \mathbb{R}_{d}[x] .
$$

For simplicity, we represent by the same symbol, say $p$, any of the three mathematical objects identified in (1). When we need to specify one of the above three sets, we will be explicit.

A positive function $g: \mathcal{M} \longrightarrow \mathbb{R}$ will be called a weight function on $\mathcal{M}$. We say that it is normalized when $g\left(\lambda_{0}\right)+g\left(\lambda_{1}\right)+\cdots+g\left(\lambda_{d}\right)=1$. We shall write, for short, $g_{l}:=g\left(\lambda_{l}\right)$. From the pair $(\mathcal{M}, g)$ we can define an inner product in $\mathbb{R}_{d}[x]$ (indistinctly in $\mathcal{F}(\mathcal{M})$ or in $\mathbb{R}[x] /(Z))$ as

$$
\langle p, q\rangle:=\sum_{l=0}^{d} g_{l} p\left(\lambda_{l}\right) q\left(\lambda_{l}\right), \quad p, q \in \mathbb{R}_{d}[x],
$$

with corresponding norm $\|\cdot\|$. From now on, this will be referred to as the scalar product associated to $(\mathcal{M}, g)$. Note that $\langle 1,1\rangle=1$ is the condition concerning the normalized character of the weight function $g$, which will be hereafter assumed.

In order to simplify some expressions, it is useful to introduce the following momentlike parameters, computed from the points of the mesh $\mathcal{M}$,

$$
\pi_{k}:=\prod_{l=0}^{d}\left|\lambda_{k}-\lambda_{l}\right|=(-1)^{k} \prod_{l=0(l \neq k)}^{d}\left(\lambda_{k}-\lambda_{l}\right) \quad(0 \leq k \leq d)
$$

and the family of interpolating polynomials (with degree $d$ ):

$$
Z_{k}:=\frac{(-1)^{k}}{\pi_{k}} \prod_{l=0(l \neq k)}^{d}\left(x-\lambda_{l}\right) \quad(0 \leq k \leq d)
$$


which satisfy:

$$
Z_{k}\left(\lambda_{h}\right)=\delta_{h k}, \quad\left\langle Z_{h}, Z_{k}\right\rangle=\delta_{h k} g_{k}
$$

Then, using Lagrange interpolation, $p=\sum_{k=0}^{d} p\left(\lambda_{k}\right) Z_{k}$ for any $p \in \mathbb{R}_{d}[x]$. In particular, when $p=x^{i}, i=0,1, \ldots, d$, we get $x^{i}=\sum_{k=0}^{d} \lambda_{k}^{i} Z_{k}$ whence, equating the terms with degree $d$,

$$
\sum_{k=0}^{d} \frac{(-1)^{k}}{\pi_{k}} \lambda_{k}^{i}=0 \quad(0 \leq i \leq d-1), \quad \sum_{k=0}^{d} \frac{(-1)^{k}}{\pi_{k}} \lambda_{k}^{d}=1 .
$$

\subsection{Forms and orthogonal systems}

Each real number $\lambda$ induces a linear form on $\mathbb{R}_{d}[x]$, defined by $[\lambda](p):=p(\lambda)$. Then, the first equality in (5) can be interpreted by saying that the forms $\left[\lambda_{0}\right],\left[\lambda_{1}\right], \ldots,\left[\lambda_{d}\right]$ are the dual basis of the polynomials $Z_{0}, Z_{1}, \ldots, Z_{d}$. The scalar product associated to $(\mathcal{M}, g)$ induces an isomorphism between the space $\mathbb{R}_{d}[x]$ and its dual, where each polynomial $p$ corresponds to the form $\omega_{p}$, defined as $\omega_{p}(q):=\langle p, q\rangle$ and, reciprocally, each form $\omega$ is associated to a polynomial $p_{\omega}$ through $\left\langle p_{\omega}, q\right\rangle=\omega(q)$. By observing how the isomorphism acts on the bases $\left\{\left[\lambda_{l}\right]\right\}_{0 \leq l \leq d},\left\{Z_{l}\right\}_{0 \leq l \leq d}$, we get the expressions:

$$
\omega_{p}=\sum_{l=0}^{d} g_{l} p\left(\lambda_{l}\right)\left[\lambda_{l}\right], \quad p_{\omega}=\sum_{l=0}^{d} \frac{1}{g_{l}} \omega\left(Z_{l}\right) Z_{l} .
$$

In particular, the polynomial corresponding to $\left[\lambda_{k}\right]$ is

$$
\begin{aligned}
H_{k} & :=p_{\left[\lambda_{k}\right]}=\sum_{l=0}^{d} \frac{1}{g_{l}}\left[\lambda_{k}\right]\left(Z_{l}\right) Z_{l}=\sum_{l=0}^{d} \frac{1}{g_{l}} \delta_{l k} Z_{l} \\
& =\frac{1}{g_{k}} Z_{k}=\frac{(-1)^{k}}{g_{k} \pi_{k}}\left(x-\lambda_{0}\right) \cdots\left(\widehat{x-\lambda_{k}}\right) \cdots\left(x-\lambda_{d}\right),
\end{aligned}
$$

and their scalar products are:

$$
\left\langle H_{h}, H_{k}\right\rangle=\left[\lambda_{h}\right]\left(H_{k}\right)=H_{k}\left(\lambda_{h}\right)=\frac{1}{g_{h}} \delta_{h k},
$$

where $\left(\widehat{x-\lambda}_{k}\right)$ denotes that this factor is not present in the product. Moreover, property (6) is equivalent to stating that the form $\sum_{k=0}^{d} \frac{(-1)^{k}}{\pi_{k}}\left[\lambda_{k}\right]$ annihilates on the space $\mathbb{R}_{d-1}[x]$.

Lemma 2.1 In the space $\mathbb{R}_{d}[x]$, let us consider the scalar product associated to $(\mathcal{M}, g)$. Then, the polynomial

$$
T:=\sum_{k=0}^{d} \frac{(-1)^{k}}{\pi_{k}} H_{k}=\sum_{k=0}^{d} \frac{1}{g_{k} \pi_{k}^{2}}\left(x-\lambda_{0}\right) \cdots\left(\widehat{x-\lambda_{k}}\right) \cdots\left(x-\lambda_{d}\right)
$$

satisfies the following: 
(a) $T$ is orthogonal to $\mathbb{R}_{d-1}[x]$;

(b) $\|T\|^{2}=\sum_{k=0}^{d} \frac{1}{g_{k} \pi_{k}^{2}}$;

(c) $T\left(\lambda_{0}\right)=\frac{1}{g_{0} \pi_{0}}$

Proof. The proof of $(a)$ is straightforward by considering the form $\omega_{T}$ associated to $T$, whereas $(b)$ and $(c)$ are proved by simple computations:

$$
\begin{aligned}
& \|T\|^{2}=\sum_{h, k=0}^{d} \frac{(-1)^{h+k}}{\pi_{h} \pi_{k}}\left\langle H_{h}, H_{k}\right\rangle=\sum_{k=0}^{d} \frac{1}{g_{k} \pi_{k}^{2}} ; \\
& T\left(\lambda_{0}\right)=\sum_{k=0}^{d} \frac{(-1)^{k}}{\pi_{k}} H_{k}\left(\lambda_{0}\right)=\frac{1}{g_{0} \pi_{0}} .
\end{aligned}
$$

A family of polynomials $r_{0}, r_{1}, \ldots, r_{d}$ is said to be an orthogonal system when each polynomial $r_{k}$ is of degree $k$ and $\left\langle r_{h}, r_{k}\right\rangle=0$ for any $h \neq k$.

Proposition 2.2 Every orthogonal system $r_{0}, r_{1}, \ldots, r_{d}$ satisfies the following properties:

(a) There exists a tridiagonal matrix $\boldsymbol{R}$ (called the recurrence matrix of the system) such that, in $\mathbb{R}[x] /(Z)$ :

$$
x \boldsymbol{r}:=x\left(\begin{array}{c}
r_{0} \\
r_{1} \\
r_{2} \\
\vdots \\
r_{d-2} \\
r_{d-1} \\
r_{d}
\end{array}\right)=\left(\begin{array}{ccccccc}
a_{0} & c_{1} & 0 & & & & \\
b_{0} & a_{1} & c_{2} & 0 & & & \\
0 & b_{1} & a_{2} & \ddots & \ddots & & \\
& 0 & \ddots & \ddots & \ddots & 0 & \\
& & \ddots & \ddots & a_{d-2} & c_{d-1} & 0 \\
& & & 0 & b_{d-2} & a_{d-1} & c_{d} \\
& & & & 0 & b_{d-1} & a_{d}
\end{array}\right)\left(\begin{array}{c}
r_{0} \\
r_{1} \\
r_{2} \\
\vdots \\
r_{d-2} \\
r_{d-1} \\
r_{d}
\end{array}\right)=\boldsymbol{R} \boldsymbol{r}
$$

and this equality, in $\mathbb{R}[x]$, reads:

$$
x \boldsymbol{r}=\boldsymbol{R} \boldsymbol{r}+\left(\begin{array}{lllll}
0 & 0 & \cdots & 0 & \frac{\left\|r_{d}\right\|^{2}}{r_{d}\left(\lambda_{0}\right)} \frac{1}{g_{0} \pi_{0}} Z
\end{array}\right)^{\top} .
$$

(b) All the entries $b_{k}, c_{k}$, of matrix $\boldsymbol{R}$ are nonzero and satisfy $b_{k} c_{k+1}>0$.

(c) The matrix $\boldsymbol{R}$ diagonalizes with eigenvalues the elements of $\mathcal{M}$. An eigenvector associated to $\lambda_{k}$ is $\left(r_{0}\left(\lambda_{k}\right), r_{1}\left(\lambda_{k}\right), \ldots, r_{d-1}\left(\lambda_{k}\right), r_{d}\left(\lambda_{k}\right)\right)^{\top}$.

(d) For every $k=1, \ldots, d$ the polynomial $r_{k}$ has real simple roots. If $\mathcal{M}_{k}$ denotes the mesh of the ordered roots of $r_{k}$, then (the points of) the mesh $\mathcal{M}_{d}$ interlaces $\mathcal{M}$ and, for each $k=1,2, \ldots, d-1, \mathcal{M}_{k}$ interlaces $\mathcal{M}_{k+1}$. 
Proof. (a) Working in $\mathbb{R}[x] /(Z)$, we have $\left\langle x r_{k}, r_{h}\right\rangle=0$ provided that $k<h-1$ and, by symmetry, the result is also zero when $h<k-1$. Therefore we can write, for any $k=0,1, \ldots, d$,

$$
x r_{k}=\sum_{h=0}^{d} \frac{\left\langle x r_{k}, r_{h}\right\rangle}{\left\|r_{h}\right\|^{2}} r_{h}=\sum_{h=\max \{0, k-1\}}^{\min \{k+1, d\}} \frac{\left\langle x r_{k}, r_{h}\right\rangle}{\left\|r_{h}\right\|^{2}} r_{h}=b_{k-1} r_{k-1}+a_{k} r_{k}+c_{k+1} r_{k+1}
$$

where, for notational convenience, we have introduced the null formal terms $b_{-1} r_{-1}$, and $c_{d+1} r_{d+1}$. Then, for any $k=0,1, \ldots, d$ the parameters $b_{k}, a_{k}, c_{k}$ are defined by:

$$
\begin{aligned}
& b_{k}=\frac{\left\langle x r_{k+1}, r_{k}\right\rangle}{\left\|r_{k}\right\|^{2}} \quad(0 \leq k \leq d-1), \quad b_{d}=0, \\
& a_{k}=\frac{\left\langle x r_{k}, r_{k}\right\rangle}{\left\|r_{k}\right\|^{2}} \quad(0 \leq k \leq d), \\
& c_{0}=0, \quad c_{k}=\frac{\left\langle x r_{k-1}, r_{k}\right\rangle}{\left\|r_{k}\right\|^{2}} \quad(1 \leq k \leq d) .
\end{aligned}
$$

Given any $k=0,1, \ldots, d$ let $Z_{k}^{*}:=\prod_{l=0, l \neq k}^{d}\left(x-\lambda_{l}\right)=\xi_{0} r_{d}+\xi_{1} r_{d-1}+\cdots$, where notice that $\xi_{0}$ does not depend on $k$. Thus,

$$
\left\langle r_{d}, Z_{k}^{*}\right\rangle=g_{k} r_{d}\left(\lambda_{k}\right)(-1)^{k} \pi_{k}=\xi_{0}\left\|r_{d}\right\|^{2}=g_{0} r_{d}\left(\lambda_{0}\right) \pi_{0} \neq 0
$$

Moreover, for any $k=0,1, \ldots, d$, we get:

$$
r_{d}-\frac{\left\|r_{d}\right\|^{2}}{r_{d}\left(\lambda_{0}\right)} \frac{1}{g_{0} \pi_{0}} Z_{k}^{*} \in \mathbb{R}_{d-1}[x], \quad \frac{r_{d}\left(\lambda_{k}\right)}{r_{d}\left(\lambda_{0}\right)}=(-1)^{k} \frac{g_{0} \pi_{0}}{g_{k} \pi_{k}} .
$$

Then, the equality $x r_{d}=b_{d-1} r_{d-1}+a_{d} r_{d}$, holding in $\mathbb{R}[x] /(Z)$, and the comparison of the degrees allows us to stablish the existence of $\psi \in \mathbb{R}$ such that $x r_{d}=b_{d-1} r_{d-1}+a_{d} r_{d}+\psi Z$ in $\mathbb{R}[x]$. Noticing that $\psi$ is the first coefficient of $r_{d}$, we get, from (10),

$$
\psi=\frac{\left\|r_{d}\right\|^{2}}{r_{d}\left(\lambda_{0}\right)} \frac{1}{g_{0} \pi_{0}}
$$

(b) By looking again to the degrees, we realize that $c_{1}, c_{2}, \ldots, c_{d}$ are nonzero. For $k=0,1, \ldots, d-1$, we have, from the equality

$$
b_{k}=\frac{\left\langle x r_{k+1}, r_{k}\right\rangle}{\left\|r_{k}\right\|^{2}}=\frac{\left\langle x r_{k}, r_{k+1}\right\rangle}{\left\|r_{k+1}\right\|^{2}} \frac{\left\|r_{k+1}\right\|^{2}}{\left\|r_{k}\right\|^{2}}=\frac{\left\|r_{k+1}\right\|^{2}}{\left\|r_{k}\right\|^{2}} c_{k+1},
$$

that the parameters $b_{0}, b_{1}, \ldots, b_{d-1}$ are also nonnull and, moreover, $b_{k} c_{k+1}>0$ for any $k=0,1, \ldots, d-1$.

(c) This result follows immediately if we evaluate, in $\mathbb{R}[x]$ and for each $\lambda_{k}$, the matrix equation obtained in $(a)$.

$(d)$ From (10) we observe that $r_{d}$ takes alternating signs on the points of $\mathcal{M}$. Hence, this polynomial has $d$ simple roots whose mesh $\mathcal{M}_{d}$ interlaces $\mathcal{M}$. Noticing that $Z$ takes 
alternating signs over the elements of $\mathcal{M}_{d}$, from the equality $b_{d-1} r_{d-1}=\left(x-a_{d}\right) r_{d}-\psi Z$ it turns out that $r_{d-1}$ takes alternating signs on the elements of $\mathcal{M}_{d}$; whence $\mathcal{M}_{d-1}$ interlaces $\mathcal{M}_{d}$ and $r_{d}$ has alternating signs on $\mathcal{M}_{d-1}$. Recursively, suppose that, for $k=1, \ldots, d-2$, the polynomials $r_{k+1}$ and $r_{k+2}$ have simple real roots and that $\mathcal{M}_{k+1}$ interlaces $\mathcal{M}_{k+2}$, so that $r_{k+2}$ takes alternating signs on $\mathcal{M}_{k+1}$. Then, the result follows by just evaluating the equality $b_{k} r_{k}=\left(x-a_{k+1}\right) r_{k+1}-c_{k+2} r_{k+2}$ at the points of $\mathcal{M}_{k+1}$.

\subsection{The canonical orthogonal system}

Consider the space $\mathbb{R}_{d}[x]$ with the scalar product associated to $(\mathcal{M}, g)$. From the identification of such a space with its dual by contraction of the scalar product, the form $\left[\lambda_{0}\right]: p \rightarrow p\left(\lambda_{0}\right)$ is represented by the polynomial $H_{0}=\frac{1}{g_{0} \pi_{0}}\left(x-\lambda_{1}\right) \cdots\left(x-\lambda_{d}\right)$ through $\left\langle H_{0}, p\right\rangle=p\left(\lambda_{0}\right)$.

For any given $0 \leq k \leq d-1$, let $q_{k} \in \mathbb{R}_{k}[x]$ denote the orthogonal projection of $H_{0}$ over $\mathbb{R}_{k}[x]$. Alternatively, the polynomial $q_{k}$ can be defined as the unique polynomial in $\mathbb{R}_{k}[x]$ such that

$$
\left\|H_{0}-q_{k}\right\|=\min \left\{\left\|H_{0}-q\right\|: q \in \mathbb{R}_{k}[x]\right\} .
$$

(See Fig. 1.) Let $\mathcal{S}$ denote the sphere in $\mathbb{R}_{d}[x]$ such that 0 and $H_{0}$ are antipodal points on it; that is, the sphere with center $\frac{1}{2} H_{0}$ and radius $\frac{1}{2}\left\|H_{0}\right\|$. Notice that its equation $\left\|p-\frac{1}{2} H_{0}\right\|^{2}=\frac{1}{4}\left\|H_{0}\right\|^{2}$ can also be written as $\|p\|^{2}=\left\langle H_{0}, p\right\rangle=p\left(\lambda_{0}\right)$. Consequently,

$$
\mathcal{S}=\left\{p \in \mathbb{R}_{d}[x]:\|p\|^{2}=p\left(\lambda_{0}\right)\right\}=\left\{p \in \mathbb{R}_{d}[x]:\left\langle H_{0}-p, p\right\rangle=0\right\} .
$$

Note also that the projection $q_{k}$ is on the sphere $\mathcal{S}_{k}:=\mathcal{S} \cap \mathbb{R}_{k}[x]$ since, in particular, $\left\langle H_{0}-q_{k}, q_{k}\right\rangle=0$.

Proposition 2.3 The polynomial $q_{k}$, which is the orthogonal projection of $H_{0}$ on $\mathbb{R}_{k}[x]$, can be defined as the unique polynomial of $\mathbb{R}_{k}[x]$ satisfying

$$
\left\langle H_{0}, q_{k}\right\rangle=q_{k}\left(\lambda_{0}\right)=\max \left\{q\left(\lambda_{0}\right): \text { for all } q \in \mathcal{S}_{k}\right\}
$$

where $\mathcal{S}_{k}$ is the sphere $\left\{q \in \mathbb{R}_{k}[x]:\|q\|^{2}=q\left(\lambda_{0}\right)\right\}$. Equivalently, $q_{k}$ is the antipodal point of the origin in $\mathcal{S}_{k}$.

Proof. Since $q_{k}$ is orthogonal to $H_{0}-q_{k}$, we have $\left\|q_{k}\right\|^{2}+\left\|H_{0}-q_{k}\right\|^{2}=\left\|H_{0}\right\|^{2}=\frac{1}{g_{0}}$. Then, as $q_{k} \in \mathcal{S}_{k}$ we get

$$
\begin{aligned}
q_{k}\left(\lambda_{0}\right) & =\left\|q_{k}\right\|^{2}=\frac{1}{g_{0}}-\left\|H_{0}-q_{k}\right\|^{2}=\frac{1}{g_{0}}-\min \left\{\left\|H_{0}-q\right\|^{2}: \text { for all } q \in \mathcal{S}_{k}\right\}= \\
& =\max \left\{\|q\|^{2}: \text { for all } q \in \mathcal{S}_{k}\right\}=\max \left\{q\left(\lambda_{0}\right): \text { for all } q \in \mathcal{S}_{k}\right\} .
\end{aligned}
$$

Considering the equivalent form $\left\|q_{k}\right\|=\max \left\{\|q\|\right.$ : for all $\left.q \in \mathcal{S}_{k}\right\}$, the proof is complete.

With the notation $q_{d}:=H_{0}$, we obtain the family of polynomials $q_{0}, q_{1}, \ldots, q_{d-1}, q_{d}$. Let us remark some of their properties. 


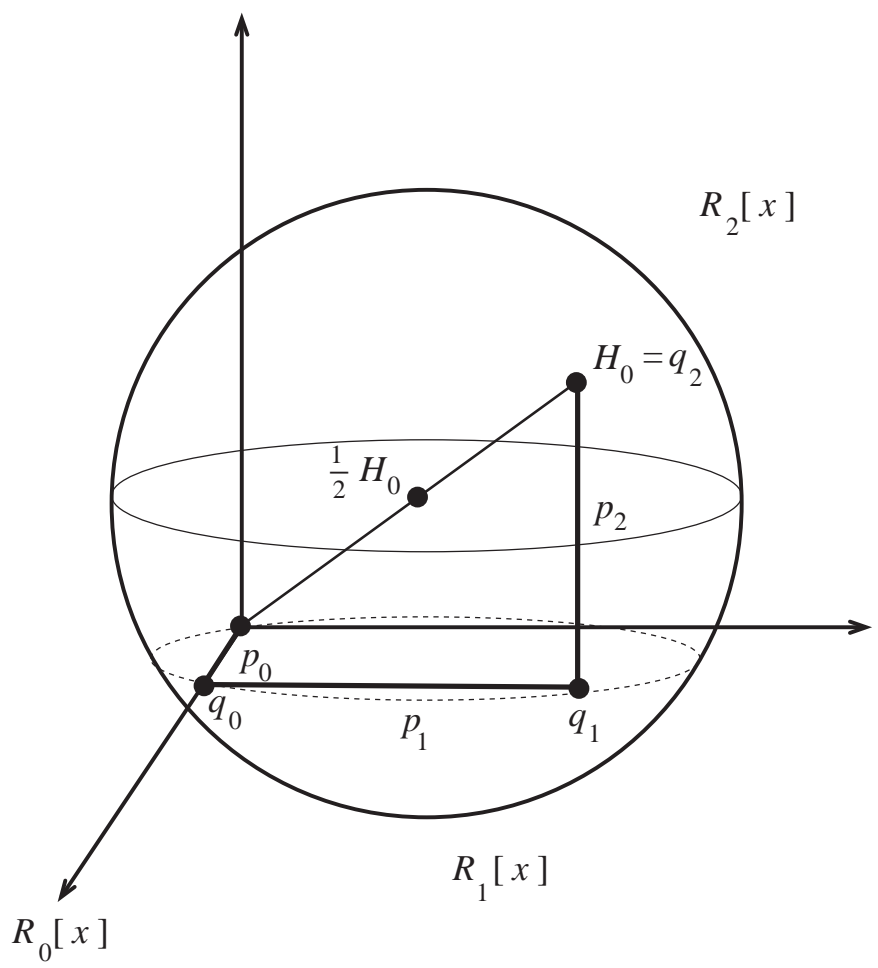

Figure 1: Obtaining the $q$ 's and the $p$ 's by projecting $H_{0}$.

Corollary 2.4 The polynomials $q_{0}, q_{1}, \ldots, q_{d-1}, q_{d}$, satisfy the following:

(a) Each $q_{k}$ has degree exactly $k$.

(b) $1=q_{0}\left(\lambda_{0}\right)<q_{1}\left(\lambda_{0}\right)<\cdots<q_{d-1}\left(\lambda_{0}\right)<q_{d}\left(\lambda_{0}\right)=\frac{1}{g_{0}}$.

(c) The polynomials $q_{0}, q_{1}, \ldots, q_{d-1}$ constitute an orthogonal system with respect to the scalar product associated to the mesh $\left\{\lambda_{1}>\lambda_{2}>\cdots>\lambda_{d}\right\}$ and the weight function $\lambda_{k} \mapsto\left(\lambda_{0}-\lambda_{k}\right) g_{k}, k=1, \ldots, d$.

Proof. (a) Notice that $\mathcal{S}_{0}=\{0,1\}$. Consequently, $q_{0}=1$. Assume that $q_{k-1}$ has degree $k-1$, but $q_{k}$ has degree lesser than $k$. Because of the uniqueness of the projection, $q_{k}=q_{k-1}$ and $H_{0}-q_{k-1}$ would be orthogonal to $\mathbb{R}_{k}[x]$. In particular,

$$
\begin{aligned}
0 & =\left\langle H_{0}-q_{k-1},\left(x-\lambda_{0}\right) q_{k-1}\right\rangle=\left\langle\left(x-\lambda_{0}\right) H_{0}-\left(x-\lambda_{0}\right) q_{k-1}, q_{k-1}\right\rangle \\
& =\left\langle\left(\lambda_{0}-x\right) q_{k-1}, q_{k-1}\right\rangle=\sum_{l=0}^{d} g_{l}\left(\lambda_{0}-\lambda_{l}\right) q_{k-1}^{2}\left(\lambda_{l}\right) .
\end{aligned}
$$

Hence, $q_{k-1}\left(\lambda_{l}\right)=0$ for any $1 \leq l \leq d$ and $q_{k-1}$ would be null.

(b) If $q_{k-1}\left(\lambda_{0}\right)=q_{k}\left(\lambda_{0}\right)$, from Proposition 2.3 we would get $q_{k-1}=q_{k}$, which is not possible because of $(a)$. 
(c) Let $0 \leq h<k \leq d-1$. Since $H_{0}-q_{k}$ is orthogonal to $\mathbb{R}_{k}[x]$ we have, in particular, that

$$
\begin{aligned}
0 & =\left\langle H_{0}-q_{k},\left(x-\lambda_{0}\right) q_{h}\right\rangle=\left\langle\left(x-\lambda_{0}\right) H_{0}-\left(x-\lambda_{0}\right) q_{k}, q_{h}\right\rangle=\left\langle\left(\lambda_{0}-x\right) q_{k}, q_{h}\right\rangle \\
& =\sum_{l=0}^{d} g_{l}\left(\lambda_{0}-\lambda_{l}\right) q_{k}\left(\lambda_{l}\right) q_{h}\left(\lambda_{l}\right)=\sum_{l=1}^{d}\left(\lambda_{0}-\lambda_{l}\right) g_{l} q_{k}\left(\lambda_{l}\right) q_{h}\left(\lambda_{l}\right)
\end{aligned}
$$

stablishing the claimed orthogonality.

The polynomial $q_{k}$, as the orthogonal projection of $H_{0}$ over $\mathbb{R}_{k}[x]$, can also be seen as the orthogonal projection of $q_{k+1}$ over $\mathbb{R}_{k}[x]$, as $q_{k+1}-q_{k}=H_{0}-q_{k}-\left(H_{0}-q_{k+1}\right)$ is orthogonal to $\mathbb{R}_{k}[x]$. Consider the family of polynomials defined as

$$
\begin{aligned}
p_{0} & :=q_{0}=1, p_{1}:=q_{1}-q_{0}, p_{2}:=q_{2}-q_{1}, \ldots, \\
p_{d-1} & :=q_{d-1}-q_{d-2}, p_{d}:=q_{d}-q_{d-1}=H_{0}-q_{d-1} .
\end{aligned}
$$

Note that, then, $q_{k}=p_{0}+p_{1}+\cdots+p_{k}(0 \leq k \leq d)$, and, in particular, $p_{0}+p_{1}+\cdots+p_{d}=H_{0}$. Let us now begin the study of the polynomials $\left(p_{k}\right)_{0 \leq k \leq d}$.

Proposition 2.5 The polynomials $p_{0}, p_{1}, \ldots, p_{d-1}, p_{d}$ constitute an orthogonal system with respect to the scalar product associated to $(\mathcal{M}, g)$.

Proof. From $p_{k}=q_{k}-q_{k-1}$ we see that $p_{k}$ has degree $k$. Moreover, we have already seen that $p_{k}=q_{k}-q_{k-1}$ is orthogonal to $\mathbb{R}_{k-1}[x]$, whence the polynomials $p_{k}$ form an orthogonal system.

The sequence of polynomials $\left(p_{k}\right)_{0 \leq k \leq d}$, defined in (11), will be called the canonical orthogonal system associated to $(\mathcal{M}, g)$. The next result gives three different characterizations of such systems.

Proposition 2.6 Let $r_{0}, r_{1}, \ldots, r_{d-1}, r_{d}$ be an orthogonal system with respect to the scalar product associated to $(\mathcal{M}, g)$. Then the following assertions are all equivalent:

(a) $\left(r_{k}\right)_{0 \leq k \leq d}$ is the canonical orthogonal system associated to $(\mathcal{M}, g)$;

(b) $r_{0}=1$ and the entries of the recurrence matrix $\boldsymbol{R}$ associated to $\left(r_{k}\right)_{0 \leq k \leq d}$, satisfy $a_{k}+b_{k}+c_{k}=\lambda_{0}$, for any $k=0,1, \ldots, d$;

(c) $r_{0}+r_{1}+\cdots+r_{d}=H_{0}$;

(d) $\left\|r_{k}\right\|^{2}=r_{k}\left(\lambda_{0}\right)$ for any $k=0,1, \ldots, d$.

Proof. Let $\left(p_{k}\right)_{0 \leq k \leq d}$ be the canonical orthogonal system associated to $(\mathcal{M}, g)$. The space $\mathbb{R}_{k}[x] \cap \mathbb{R}_{k-1}^{\perp}[x]$ has dimension one, and hence the polynomials $r_{k}, p_{k}$ are proportional: $r_{k}=\xi_{k} p_{k}$. Let $\boldsymbol{j}:=\left(\begin{array}{llll}1 & 1 & \cdots & 1\end{array}\right)^{\top}$. 
$(a) \Rightarrow(b)$ : We have $r_{0}=p_{0}=1$. Consider the recurrence matrix $\boldsymbol{R}$ (Proposition 2.2) associated to the canonical orthogonal system $\left(r_{k}\right)_{0 \leq k \leq d}=\left(p_{k}\right)_{0 \leq k \leq d}$. Then, computing $x q_{d}$ in $\mathbb{R}[x] /(Z)$ in two different ways we get:

$$
\begin{aligned}
x q_{d} & =x \sum_{k=0}^{d} p_{k}=x \boldsymbol{j}^{\top} \boldsymbol{p}=\boldsymbol{j}^{\top} \boldsymbol{R} \boldsymbol{p}=\left(\begin{array}{llll}
a_{0}+b_{0} & c_{1}+a_{1}+b_{1} & \cdots & c_{d}+a_{d}
\end{array}\right)^{\top} \boldsymbol{p} \\
& =\sum_{k=0}^{d}\left(a_{k}+b_{k}+c_{k}\right) p_{k} ; \\
x q_{d} & =x H_{0}=\lambda_{0} H_{0}=\sum_{k=0}^{d} \lambda_{0} p_{k},
\end{aligned}
$$

and, from the linear independence of the polynomials $p_{k}$, we get $a_{k}+b_{k}+c_{k}=\lambda_{0}$.

$(b) \Rightarrow(c)$ : Working in $\mathbb{R}[x] /(Z)$ and from $x \boldsymbol{r}=\boldsymbol{R} \boldsymbol{r}$, we have:

$$
0=\boldsymbol{j}^{\top}(x \boldsymbol{r}-\boldsymbol{R} \boldsymbol{r})=x \sum_{k=0}^{d} r_{k}-\boldsymbol{j}^{\top} \boldsymbol{R} \boldsymbol{r}=x \sum_{k=0}^{d} r_{k}-\lambda_{0} \boldsymbol{j}^{\top} \boldsymbol{r}=\left(x-\lambda_{0}\right) \sum_{k=0}^{d} r_{k} .
$$

Therefore there exists $\xi$ such that $\sum_{k=0}^{d} r_{k}=\xi H_{0}=\sum_{k=0}^{d} \xi p_{k}$. Since, also, $\sum_{k=0}^{d} r_{k}=$ $\sum_{k=0}^{d} \xi_{k} p_{k}$, where $\xi_{0}=1$, it turns out that $\xi_{0}=\xi_{1}=\cdots=\xi_{d}=\xi=1$. Consequently, $\sum_{k=0}^{d} r_{k}=H_{0}$.

$(c) \Rightarrow(d):\left\|r_{k}\right\|^{2}=\left\langle r_{k}, r_{0}+r_{1}+\cdots+r_{d}\right\rangle=\left\langle r_{k}, H_{0}\right\rangle=r_{k}\left(\lambda_{0}\right)$.

$(d) \Rightarrow(a)$ : From $r_{k}=\xi_{k} p_{k}$, we have $\xi_{k}^{2}\left\|p_{k}\right\|^{2}=\left\|r_{k}\right\|^{2}=r_{k}\left(\lambda_{0}\right)=\xi_{k} p_{k}\left(\lambda_{0}\right)=\xi_{k}\left\|p_{k}\right\|^{2}$. Whence $\xi_{k}=1$ and $r_{k}=p_{k}$.

Corollary 2.7 The highest degree polynomial $p_{d}$ of the canonical orthogonal system associated with $(\mathcal{M}, g)$ satisfies the following:

(a) $p_{d}=\left(\sum_{l=0}^{d} \frac{g_{0} \pi_{0}}{g_{l} \pi_{l}^{2}}\right)^{-1} \sum_{k=0}^{d} \frac{1}{g_{k} \pi_{k}^{2}}\left(x-\lambda_{0}\right) \cdots\left(\widehat{x-\lambda_{k}}\right) \cdots\left(x-\lambda_{d}\right)$;

(b) $p_{d}\left(\lambda_{0}\right)=\frac{1}{g_{0}}\left(\sum_{l=0}^{d} \frac{g_{0} \pi_{0}^{2}}{g_{l} \pi_{l}^{2}}\right)^{-1} ; \quad p_{d}\left(\lambda_{k}\right)=(-1)^{k} \frac{g_{0} \pi_{0}}{g_{k} \pi_{k}} p_{d}\left(\lambda_{0}\right) \quad(1 \leq k \leq d)$.

Proof. Recalling that the polynomial $T=\sum_{k=0}^{d} \frac{(-1)^{k}}{\pi_{k}} H_{k}$, introduced in Lemma 2.1, is orthogonal to $\mathbb{R}_{d-1}[x]$, there exists a constant $\xi$ such that $p_{d}=\xi T$. From $\left\|p_{d}\right\|^{2}=p_{d}\left(\lambda_{0}\right)$, we then obtain $p_{d}=\frac{T\left(\lambda_{0}\right)}{\|T\|^{2}} T$. Substituting into this formula the values of $T\left(\lambda_{0}\right)$ and $\|T\|^{2}$, given also in Lemma 2.1, we obtain the claimed expressions for $p_{d}, p_{d}\left(\lambda_{0}\right)$ and $p_{d}\left(\lambda_{k}\right)$.

From the last equality of Corollary 2.7 we get:

$$
g_{k}=g_{0} \frac{\pi_{0}}{\pi_{k}} \frac{p_{d}\left(\lambda_{0}\right)}{(-1)^{k} p_{d}\left(\lambda_{k}\right)} \quad(0 \leq k \leq d)
$$

which, together with the normalization of $g$, implies that, given $\mathcal{M}$, the knowledge of $p_{d}$ allows us to reconstruct the weight function. 


\subsection{The conjugate canonical orthogonal system}

Consider a given mesh $\mathcal{M}=\left\{\lambda_{0}>\lambda_{1}>\cdots>\lambda_{d}\right\}$. As we have seen, each normalized weight function $g: \mathcal{M} \rightarrow \mathbb{R}$ induces a scalar product and its corresponding canonical orthogonal system $\left(p_{k}\right)_{0 \leq k \leq d}$. Moreover, we know that its recurrence matrix $\boldsymbol{R}$, given in Proposition 2.2, satisfies:

$$
x \boldsymbol{p}=\boldsymbol{R} \boldsymbol{p}, \quad \boldsymbol{j}^{\top} \boldsymbol{R}=\lambda_{0} \boldsymbol{j}^{\top},
$$

where $\boldsymbol{p}$ and $\boldsymbol{j}$ are the column matrices $\left(\begin{array}{llll}p_{0} & p_{1} & \cdots & p_{d}\end{array}\right)^{\top}$ and $\left(\begin{array}{llll}1 & 1 & \cdots & 1\end{array}\right)^{\top}$, respectively.

Given an $n \times m$ matrix $\boldsymbol{A}=\left(a_{i j}\right)$ we denote by $\boldsymbol{A}^{\star}$ the $n \times m$ matrix with $(i, j)$-entry $a_{n-i+1, m-j+1}$, which results by applying a central symmetry to $\boldsymbol{A}$. It is immediate to check that $(\lambda \boldsymbol{A}+\mu \boldsymbol{B})^{\star}=\lambda \boldsymbol{A}^{\star}+\mu \boldsymbol{B}^{\star}$ and $(\boldsymbol{A} \boldsymbol{B})^{\star}=\boldsymbol{A}^{\star} \boldsymbol{B}^{\star}$. The square $(d+1)$-matrix $\boldsymbol{S}$ with null entries excepting those on the principal antidiagonal which are 1's, satisfies $\boldsymbol{S}^{\top}=\boldsymbol{S}^{-1}=\boldsymbol{S}$ and, when $\boldsymbol{A}$ is any square $(d+1)$-matrix, $\boldsymbol{A}^{\star}=\boldsymbol{S} \boldsymbol{A} \boldsymbol{S}$.

The polynomial $p_{d}$ has an inverse in $\mathbb{R}[x] /(Z)$ and, therefore, we can define $\tilde{p}_{k}:=$ $p_{d}^{-1} p_{d-k}$ for any $k=0,1, \ldots, d$. Then, with the notation

$$
\tilde{\boldsymbol{p}}:=\left(\begin{array}{llll}
\tilde{p}_{0} & \tilde{p}_{1} & \cdots & \tilde{p}_{d}
\end{array}\right)^{\top}=p_{d}^{-1} \boldsymbol{p}^{\star}=\left(\begin{array}{llll}
p_{d}^{-1} p_{d} & p_{d}^{-1} p_{d-1} & \cdots & p_{d}^{-1} p_{0}
\end{array}\right)^{\top}
$$

we obtain, from (13),

$$
x \tilde{\boldsymbol{p}}=\boldsymbol{R}^{\star} \tilde{\boldsymbol{p}}, \quad \boldsymbol{j}^{\top} \boldsymbol{R}^{\star}=\lambda_{0} \boldsymbol{j}^{\top} .
$$

The entries of the tridiagonal matrix $\boldsymbol{R}^{\star}$, which, according to the notation of Proposition 2.2 , are denoted by $\tilde{a}_{k}, \tilde{b}_{k}, \tilde{c}_{k}$, are defined by: $\tilde{a}_{k}=a_{d-k}, \tilde{b}_{k}=c_{d-k}, \tilde{c}_{k}=b_{d-k}$ and $\tilde{a}_{k}+\tilde{b}_{k}+\tilde{c}_{k}=\lambda_{0}$, for $k=0,1, \ldots, d$. Since $\tilde{b}_{k} \tilde{c}_{k+1}=c_{d-k} b_{d-k-1}>0$ and $\tilde{p}_{0}=1$ it turns out that each $\tilde{p}_{k}$ has degree $k$.

Let $\boldsymbol{P}$, respectively $\tilde{\boldsymbol{P}}$, denote the square $(d+1)$-matrix with $(i, j)$-entry $p_{i}\left(\lambda_{j}\right)$, respectively $\tilde{p}_{i}\left(\lambda_{j}\right), 0 \leq i, j \leq d$. Also, let us consider the following diagonal matrices $\boldsymbol{D}:=\operatorname{diag}\left(\left\|p_{0}\right\|^{2},\left\|p_{1}\right\|^{2}, \ldots,\left\|p_{d}\right\|^{2}\right), \tilde{\boldsymbol{D}}:=\operatorname{diag}\left(\tilde{p}_{0}\left(\lambda_{0}\right), \tilde{p}_{1}\left(\lambda_{0}\right), \ldots, \tilde{p}_{d}\left(\lambda_{0}\right)\right), \boldsymbol{P}_{d}:=$ $\operatorname{diag}\left(p_{d}\left(\lambda_{0}\right), p_{d}\left(\lambda_{1}\right), \ldots, p_{d}\left(\lambda_{d}\right)\right)$, and $\boldsymbol{G}:=\operatorname{diag}\left(g_{0}, g_{1}, \ldots, g_{d}\right)$. Now, we have the following facts:

(a) The sequence $\left(p_{k}\right)_{0 \leq k \leq d}$ is the canonical orthogonal system with respect to the inner product associated to $(\mathcal{M}, g)$ if and only if $\operatorname{dgr} p_{k}=k, 0 \leq k \leq d$, and

$$
\boldsymbol{P G P} \boldsymbol{P}^{\top}=\boldsymbol{D}
$$

(b) By the definition of $\tilde{\boldsymbol{P}}$ we immediately have $\tilde{\boldsymbol{P}}=\boldsymbol{S} \boldsymbol{P} \boldsymbol{P}_{d}^{-1}$;

(c) Similarly, from the definition of $\tilde{\boldsymbol{D}}$, we get $\tilde{\boldsymbol{D}}=p_{d}^{-1}\left(\lambda_{0}\right) \boldsymbol{S} \boldsymbol{D} \boldsymbol{S}$.

Then, the following computation

$$
\begin{aligned}
\tilde{\boldsymbol{P}}\left(p_{d}^{-1}\left(\lambda_{0}\right) \boldsymbol{P}_{d} \boldsymbol{G} \boldsymbol{P}_{d}\right) \tilde{\boldsymbol{P}}^{\top} & =p_{d}^{-1}\left(\lambda_{0}\right) \tilde{\boldsymbol{P}} \boldsymbol{P}_{d} \boldsymbol{G} \boldsymbol{P}_{d} \tilde{\boldsymbol{P}}^{\top} \\
& =p_{d}^{-1}\left(\lambda_{0}\right) \boldsymbol{S} \boldsymbol{P} \boldsymbol{P}_{d}^{-1} \boldsymbol{P}_{d} \boldsymbol{G} \boldsymbol{P}_{d} \boldsymbol{P}_{d}^{-1} \boldsymbol{P}^{\top} \boldsymbol{S} \\
& =p_{d}^{-1}\left(\lambda_{0}\right) \boldsymbol{S} \boldsymbol{P} \boldsymbol{G} \boldsymbol{P}^{\top} \boldsymbol{S}=p_{d}^{-1}\left(\lambda_{0}\right) \boldsymbol{S} \boldsymbol{D} \boldsymbol{S}=\tilde{\boldsymbol{D}},
\end{aligned}
$$


establishes that the family of polynomials $\left(\tilde{p}_{k}\right)_{0 \leq k \leq d}$, with $\operatorname{dgr} \tilde{p}_{k}=k, 0 \leq k \leq d$, is the canonical orthogonal system with respect to the product $(\mathcal{M}, \tilde{g})$, where, using Corollary 2.7, $\tilde{g}_{k}:=\tilde{g}\left(\lambda_{k}\right)$ corresponds to the expression:

$$
\begin{aligned}
\tilde{g}_{k} & =p_{d}^{-1}\left(\lambda_{0}\right)\left(\boldsymbol{P}_{d} \boldsymbol{G} \boldsymbol{P}_{d}\right)_{k k}=p_{d}^{-1}\left(\lambda_{0}\right) g_{k} p_{d}^{2}\left(\lambda_{k}\right) \\
& =g_{0} \frac{g_{0} \pi_{0}^{2}}{g_{k} \pi_{k}^{2}} p_{d}\left(\lambda_{0}\right)=\frac{g_{0} \pi_{0}^{2}}{g_{k} \pi_{k}^{2}}\left(\sum_{l=0}^{d} \frac{g_{0} \pi_{0}^{2}}{g_{l} \pi_{l}^{2}}\right)^{-1} .
\end{aligned}
$$

Note that, in particular, $\tilde{g}: \mathcal{M} \rightarrow \mathbb{R}$ is a normalized weight function on $\mathcal{M}$. All the above facts are summarized in the following result:

Proposition 2.8 Given a mesh $\mathcal{M}=\left\{\lambda_{0}>\lambda_{1}>\cdots>\lambda_{d}\right\}$, we associate, to each normalized weight function $g: \mathcal{M} \rightarrow \mathbb{R}$, a new weight function $\tilde{g}: \mathcal{M} \rightarrow \mathbb{R}$, which is also normalized, defined as:

$$
\tilde{g}_{k}=\frac{g_{0} \pi_{0}^{2}}{g_{k} \pi_{k}^{2}}\left(\sum_{l=0}^{d} \frac{g_{0} \pi_{0}^{2}}{g_{l} \pi_{l}^{2}}\right)^{-1} \quad(0 \leq k \leq d) .
$$

Then the respective canonical orthogonal systems:

$\left(p_{k}\right)_{0 \leq k \leq d}$ with respect to $\langle p, q\rangle=\sum_{l=0}^{d} g_{l} p\left(\lambda_{l}\right) q\left(\lambda_{l}\right)$; and

$\left(\tilde{p}_{k}\right)_{0 \leq k \leq d}$ with respect to $\langle p, q\rangle^{\tilde{2}}:=\sum_{l=0}^{d} \tilde{g}_{l} p\left(\lambda_{l}\right) q\left(\lambda_{l}\right)$;

are related by $\tilde{p}_{k}=p_{d}^{-1} p_{d-k}, 0 \leq k \leq d$, and the respective recurrence matrices, $\boldsymbol{R}$ and $\tilde{\boldsymbol{R}}$, coincide up to a central symmetry.

Corollary 2.9 The mapping $g \mapsto \tilde{g}$, defined on the set of normalized weight functions on $\mathcal{M}$, is involutive.

Proof. The result follows immediately from the fact that $\tilde{\boldsymbol{R}}$ is the matrix obtained by applying a central symmetry to $\boldsymbol{R}$. Or, alternatively, since $\tilde{p}_{d}=p_{d}^{-1}$ we have: $\tilde{p}_{d}^{-1} \tilde{p}_{d-k}=$ $p_{d} p_{d}^{-1} p_{k}=p_{k}$. Whence, using (12), it turns out that the conjugate weight function of $\tilde{g}$ is $g$ itself.

We shall say that the weight functions $g$ and $\tilde{g}$, the respective scalar products, and the corresponding canonical orthogonal systems are mutually conjugate.

\subsection{The dual canonical polynomials}

Associated to the canonical polynomials, there is another set of orthogonal polynomials, which are called the "dual (canonical) polynomials". In order to introduce them, notice that the orthogonality property in (15) can also be written as $\boldsymbol{P}^{\top} \boldsymbol{D}^{-1} \boldsymbol{P}=\boldsymbol{G}^{-1}$. This may be rewritten, in turn, as

$$
\widehat{\boldsymbol{P}} \boldsymbol{D} \widehat{\boldsymbol{P}}^{\top}=\boldsymbol{G}^{-1}
$$


where we have introduced the new matrix $\widehat{\boldsymbol{P}}:=\boldsymbol{P}^{\top} \boldsymbol{D}^{-1}$. Then, note that (17) can also be interpreted as an orthogonality property, with respect to the scalar product

$$
\langle p, q\rangle^{*}:=\sum_{l=0}^{d}\left\|p_{l}\right\|^{2} p\left(\lambda_{l}\right) q\left(\lambda_{l}\right)
$$

for the new polynomials $\hat{p}_{k}, 0 \leq k \leq d$, defined as

$$
\hat{p}_{k}\left(\lambda_{l}\right):=(\widehat{\boldsymbol{P}})_{k l}=\frac{p_{l}\left(\lambda_{k}\right)}{\left\|p_{l}\right\|^{2}}=\frac{p_{l}\left(\lambda_{k}\right)}{p_{l}\left(\lambda_{0}\right)} \quad(0 \leq l \leq d),
$$

and which will be called the dual polynomials of the $p_{k}$. Thus, (17) reads

$$
\left\langle\hat{p}_{k}, \hat{p}_{l}\right\rangle^{*}=\delta_{k l} g_{l}^{-1} \quad(0 \leq k, l \leq d),
$$

whence, using (18), the values of the weight function can be computed from the polynomials $\left(p_{k}\right)_{0 \leq k \leq d}$ as:

$$
g_{l}=\frac{1}{\left(\left\|\hat{p}_{l}\right\|^{*}\right)^{2}}=\left(\sum_{k=0}^{d} \frac{p_{k}\left(\lambda_{l}\right)^{2}}{p_{k}\left(\lambda_{0}\right)}\right)^{-1} .
$$

This is an alternative formula to (12).

Moreover, we have already seen in Proposition 2.2(c) that the $l$-th column of $\boldsymbol{P}$, namely $\left(p_{0}\left(\lambda_{l}\right), p_{1}\left(\lambda_{l}\right), \ldots, p_{d}\left(\lambda_{l}\right)\right)^{\top}$, is an eigenvector of the tridiagonal recurrence matrix $\boldsymbol{R}$, with eigenvalue $\lambda_{l}$. That is,

$$
\boldsymbol{R P}=\boldsymbol{P} \boldsymbol{D}_{\lambda}
$$

where $\boldsymbol{D}_{\lambda}:=\operatorname{diag}\left(\lambda_{0}, \lambda_{1}, \ldots, \lambda_{d}\right)$. Similarly, from (15) and the definition of $\widehat{\boldsymbol{P}}$ we see that $\boldsymbol{P}^{-1}=\boldsymbol{G} \boldsymbol{P}^{\top} \boldsymbol{D}^{-1}=\boldsymbol{G} \widehat{\boldsymbol{P}}$. Then, (22) yields

$$
\widehat{\boldsymbol{P}} \boldsymbol{R}=\boldsymbol{D}_{\lambda} \widehat{\boldsymbol{P}}
$$

That is, the $k$-th row of $\widehat{\boldsymbol{P}}$,

$$
\left(\hat{p}_{k}\left(\lambda_{0}\right), \hat{p}_{k}\left(\lambda_{1}\right), \ldots, \hat{p}_{k}\left(\lambda_{d}\right)\right)=\left(\frac{p_{0}\left(\lambda_{k}\right)}{p_{0}\left(\lambda_{0}\right)}, \frac{p_{1}\left(\lambda_{k}\right)}{p_{1}\left(\lambda_{0}\right)}, \ldots, \frac{p_{d}\left(\lambda_{k}\right)}{p_{d}\left(\lambda_{0}\right)}\right),
$$

is a left eigenvector of $\boldsymbol{R}$ with eigenvalue $\lambda_{k}$.

The number of sign-changes in a given sequence of real numbers is the number of times that consecutive terms (after removing the null ones) have distinct sign. Thus, if $\left(p_{k}\right)_{0 \leq k \leq d}$ is a (canonical) orthogonal system, the fact that $\operatorname{dgr} p_{k}=k$ implies that the sequence $p_{k}\left(\lambda_{0}\right), p_{k}\left(\lambda_{1}\right), \ldots, p_{k}\left(\lambda_{d}\right)$ has exactly $k$ sign-changes. Although the degrees of the dual polynomials $\left(\hat{p}_{k}\right)_{0 \leq k \leq d}$ do not necessarily coincide with their indexes, they keep the above property and the sequence $\hat{p}_{k}\left(\lambda_{0}\right), \hat{p}_{k}\left(\lambda_{1}\right), \ldots, \hat{p}_{k}\left(\lambda_{d}\right)$ also has exactly $k$ signchanges. This is a direct consequence of a known result about orthogonal polynomials (see e.g. $[27,24]$ ), which we formally state in the the next lemma, and prove it by considering the "equivalent" sequence $p_{0}\left(\lambda_{l}\right), p_{1}\left(\lambda_{l}\right), \ldots, p_{d}\left(\lambda_{l}\right)$ (since $p_{k}\left(\lambda_{0}\right)>0$ for any $0 \leq k \leq d)$ ) 


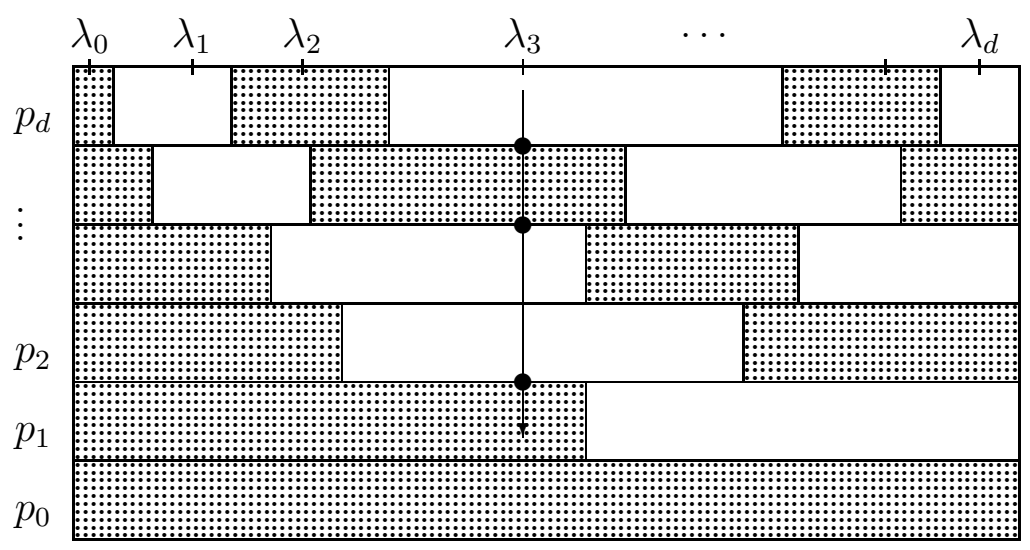

Figure 2: Proof of Lemma 2.10.

Lemma 2.10 Let $\left(p_{k}\right)_{0 \leq k \leq d}$ be a sequence of orthogonal polynomials and let $\lambda_{0}>\lambda_{1}>$ $\cdots>\lambda_{d}$ be the zeros of $p_{d+1}$. Then, for every $0 \leq l \leq d$, the sequence $p_{0}\left(\lambda_{l}\right), p_{1}\left(\lambda_{l}\right), \ldots$, $p_{d}\left(\lambda_{l}\right)$ has exactly l sign-changes.

Proof. We know that, between any two consecutive zeros of $p_{k+1}$, there lies one zero of $p_{k}$. With this in mind, this could be seen as a "proof without words"; consider Fig. 2: The number of sign-changes coincide with the crossed "staircases".

A "non-visual" proof of this result can be found in Godsil [24]. Moreover, since each column of the recurrence matrix sums to $\lambda_{0}$, we also have the following corollary:

Corollary 2.11 Let us consider a recurrence with coefficients satisfying $a_{k}+b_{k}+c_{k}=\lambda_{0}$, $0 \leq k \leq d$. Then, for every $1 \leq l \leq d$, the sequence $\hat{p}_{l}\left(\lambda_{0}\right)-\hat{p}_{l}\left(\lambda_{1}\right), \ldots, \hat{p}_{l}\left(\lambda_{d-1}\right)-\hat{p}_{l}\left(\lambda_{d}\right)$ has exactly $l-1$ sign-changes.

Proof. Let $\boldsymbol{C}$ be the $(d+1) \times(d+1)$ matrix with 1's on the principal diagonal, -1 's on the diagonal below the principal one, and 0's elsewhere. We know that $\widehat{\boldsymbol{P}}_{l}:=$ $\left(\hat{p}_{l}\left(\lambda_{0}\right), \hat{p}_{l}\left(\lambda_{1}\right), \ldots, \hat{p}_{l}\left(\lambda_{d}\right)\right)$ is a (left) eigenvector of the recurrence matrix $\boldsymbol{R}$, so that $\widehat{\boldsymbol{P}}_{l} \boldsymbol{C}$ is an eigenvector of the (also tridiagonal) matrix $\boldsymbol{R}^{\prime}:=\boldsymbol{C}^{-1} \boldsymbol{R} \boldsymbol{C}$. From this, one deduces that $\left(\hat{p}_{l}\left(\lambda_{0}\right)-\hat{p}_{l}\left(\lambda_{1}\right), \ldots, \hat{p}_{l}\left(\lambda_{d-1}\right)-\hat{p}_{l}\left(\lambda_{d}\right)\right)$ is a left eigenvector of the $d \times d$ principal submatrix of $\boldsymbol{R}^{\prime}$ :

$$
\left(\begin{array}{ccccc}
\lambda_{0}-b_{0}-c_{1} & c_{1} & & & \\
b_{1} & \lambda_{0}-b_{1}-c_{2} & c_{2} & & \\
& b_{2} & \cdot & & \\
& & \cdot & \cdot & \\
& & & \cdot & \cdot \\
& & & b_{d-1} & \lambda_{0}-b_{d-1}-c_{d}
\end{array}\right)
$$

with corresponding eigenvalue $\lambda_{l} \in$ ev $\boldsymbol{R} \backslash\left\{\lambda_{0}\right\}$. Then the result follows from Lemma 2.10 . 


\section{Applications to graphs and codes}

This section is devoted to discuss some applications of the results about orthogonal polynomials given in Section 1 to the area of algebraic graph theory. We are specially interested in the notion of distance-regularity, defined in Subsection 3.3, and we will survey some results obtained from the study of two different meshes and their corresponding orthogonal canonical systems: the local spectrum of a given graph $G$, defined in Subsection 3.2, and the spectrum of a subset of its vertex set, defined in Subsection 3.7.

Throughout this section $G=(V, E)$ denotes a simple undirected graph with vertex set $V=\{1,2, \ldots, n\}$ and edge set $E$. Two vertices $i \in V$ and $j \in V$ are adjacent if $\{i, j\} \in E$ and we denote it by $i \sim j$. A walk of length $k$ from $i \in V$ to $j \in V$ is a finite sequence of vertices of $G, i=u_{0}, u_{1}, u_{2}, \ldots, u_{k}=j$, such that $u_{t} \sim u_{t+1}, 0 \leq t \leq k-1$, and the minimum length of such a walk is the distance $\operatorname{dist}(i, j)$ between $i$ and $j$. If there is no walk from $i$ to $j$ we write $\operatorname{dist}(i, j)=\infty$. The $k$-apart from a vertex $i, \Gamma_{k}(i)$, is the set of vertices at distance $k$ from $i$, and the $k$-neighbourhood of $i, N_{k}(i)$, is the set of vertices at distance at most $k$ from $i$, that is, $N_{k}(i)=\Gamma_{0}(i) \cup \Gamma_{1}(i) \cup \cdots \cup \Gamma_{k}(i)$. The degree of a vertex $i$ is $\delta_{i}=\left|\Gamma_{1}(i)\right|$ and its eccentricity is $\operatorname{ecc}(i)=\max _{1 \leq j \leq n} \operatorname{dist}(i, j)$. Finally, the diameter of $G$ is $D=D(G)=\max _{1 \leq i \leq n} \operatorname{ecc}(i)$ and its radius $r=r(G)=\min _{1 \leq i \leq n} \operatorname{ecc}(i)$. Notice that the diameter can be equivalently defined as the maximum distance between two vertices and $G$ is connected if and only if $D(G)<\infty$.

\subsection{Some algebraic results about graphs}

One of the most important tools in the study of the algebraic properties of a graph $G$ is its adjacency matrix, $\boldsymbol{A}=\boldsymbol{A}(G)$, which is the $n \times n$ matrix with entries

$$
(\boldsymbol{A})_{i j}= \begin{cases}1 & \text { if } i \sim j \\ 0 & \text { otherwise }\end{cases}
$$

Since $G$ is taken to be a simple undirected graph, $\boldsymbol{A}(G)$ is a symmetric $(0,1)$-matrix with zero diagonal entries. We denote the characteristic polynomial of $\boldsymbol{A}(G)$ by $\phi_{G}(x)$ (since it is uniquely determined by the underlying graph $G$ ) and we will refer to it as the characteristic polynomial of $G$. The spectrum of $G$ is the set of eigenvalues of $\boldsymbol{A}(G)$ together with their multiplicities, and we will write

$$
\operatorname{sp} G:=\operatorname{sp} \boldsymbol{A}=\left\{\lambda_{0}^{m\left(\lambda_{0}\right)}, \lambda_{1}^{m\left(\lambda_{1}\right)}, \ldots, \lambda_{d}^{m\left(\lambda_{d}\right)}\right\},
$$

where $\lambda_{0}>\lambda_{1}>\ldots>\lambda_{d}$ and $m\left(\lambda_{l}\right)$ is the multiplicity of $\lambda_{l}$ as a root of $\phi_{G}(x)$. Similarly,

$$
\text { ev } G:=\left\{\lambda_{0}, \lambda_{1}, \ldots, \lambda_{d}\right\}
$$

denotes the set of different eigenvalues of $G$. If we consider matrices and vectors indexed by the vertices of $G$ and associate to any vertex $i \in V$ the $i$-th unitary vector $\boldsymbol{e}_{i}$ of the canonical basis of $\mathbb{R}^{n}$, we can interpret the adjacency matrix $\boldsymbol{A}$ as an endomorphism of $\mathbb{R}^{n}$, and the following equivalence holds:

$$
\boldsymbol{A u}=\lambda \boldsymbol{u} \quad \Longleftrightarrow \quad \sum_{j \sim i} u_{j}=\lambda u_{i} \quad(1 \leq i \leq n) .
$$


Moreover, taking the mesh $\mathcal{M}=$ ev $G$ and proceeding as in (3) we can consider the moment-like parameters

$$
\pi_{l}:=\prod_{h=0, h \neq l}^{d}\left|\lambda_{l}-\lambda_{h}\right| \quad(0 \leq l \leq d)
$$

satisfying (6):

$$
\sum_{l=0}^{d}(-1)^{l} \frac{\lambda_{l}^{k}}{\pi_{l}}= \begin{cases}0 & \text { if } 0 \leq k<d \\ 1 & \text { if } k=d .\end{cases}
$$

When $G$ is connected, the Perron-Frobenius Theorem for nonnegative matrices guaranties that its largest eigenvalue, $\lambda_{0}$, is positive and simple and has an eigenvector $\boldsymbol{v}=\left(v_{1}, \ldots, v_{n}\right)$ with all its components positive. In particular we can normalize $\boldsymbol{v}$ in such a way that $\min _{1 \leq i \leq n} v_{i}=1$. Given $U$ a subset of the vertices of $G$, let $\boldsymbol{\rho}$ be the mapping defined by $\boldsymbol{\rho} U:=\sum_{i \in U} v_{i} \boldsymbol{e}_{i}$. In what follows we suppose, for simplicity, that $G$ is connected and $\delta$-regular, that is $\delta_{i}=\delta$ for every $i \in V$. In this case, $\lambda_{0}=\delta$ and $\boldsymbol{v}=\boldsymbol{j}$, the all-1 vector, and $\boldsymbol{\rho} U$ turns out to be the characteristic vector of $U$, that is, $(\boldsymbol{\rho} U)_{i}=1$ if $i \in U$ and $(\rho U)_{i}=0$ otherwise.

The adjacency algebra $\mathcal{A}(G)$ of $G$ is the algebra of polynomials in its adjacency matrix $\boldsymbol{A}$. A simple inductive argument proves that the number of walks of length $k$ between vertices $i$ and $j$ is $\left(\boldsymbol{A}^{k}\right)_{i j}$ (see [2] for example). Therefore, if $G$ has diameter $D$, then the set $\left\{\boldsymbol{I}, \boldsymbol{A}, \boldsymbol{A}^{2}, \ldots, \boldsymbol{A}^{D}\right\}$ is linearly independent in $\mathcal{A}(G)$, or to put it in another way, the dimension of $\mathcal{A}(G)$ is at least $D+1$. Moreover, if $G$ has $d+1$ distinct eigenvalues, the minimum polynomial of $\boldsymbol{A}$ has degree $d$ and the dimension of $\mathcal{A}(G)$ is $d+1$. This fact gives us an upper bound for the diameter of a connected graph $G$, namely $D \leq d=\mid$ ev $G \mid-1$. When the equality holds, the diameter is spectrally maximal and we say that $G$ is extremal.

\subsection{Local spectrum}

For each eigenvalue $\lambda_{l} \in \operatorname{sp} G$, let $\boldsymbol{U}_{l}$ be the matrix whose columns form an orthogonal basis for the eigenspace $\mathcal{E}_{l}:=\operatorname{Ker}\left(\boldsymbol{A}-\lambda_{l} \boldsymbol{I}\right)$ associated to $\lambda_{l}$. The principal idempotents of $\boldsymbol{A}$ are the matrices $\boldsymbol{E}_{l}:=\boldsymbol{U}_{l} \boldsymbol{U}_{l}^{\top}$ representing the orthogonal projections onto $\mathcal{E}_{l}$. They can be obtainded by the formula $\boldsymbol{E}_{l}=Z_{l}(\boldsymbol{A}), 0 \leq l \leq d$, where $Z_{l}$ is the interpolating polynomial in (4) and $d=\mid$ ev $G \mid-1$. In particular, $\boldsymbol{E}_{0}=\left(\boldsymbol{v} \boldsymbol{v}^{\top}\right) /\|\boldsymbol{v}\|^{2}$. These matrices satisfy the following properties (see [24]):

(a) $\boldsymbol{E}_{l} \boldsymbol{E}_{h}= \begin{cases}\boldsymbol{E}_{l}, & \text { if } l=h \\ \mathbf{0}, & \text { otherwise; }\end{cases}$

(b) $\boldsymbol{A} \boldsymbol{E}_{l}=\lambda_{l} \boldsymbol{E}_{l}$;

(c) $p(\boldsymbol{A})=\sum_{l=0}^{d} p\left(\lambda_{l}\right) \boldsymbol{E}_{l}$, for any $p \in \mathbb{R}[x]$. 
By taking $p=1$ in $(c)$ we obtain $\sum_{l=0}^{d} \boldsymbol{E}_{l}=\boldsymbol{I}$, and for $p=x$ we get the so-called Spectral Decomposition Theorem:

$$
\boldsymbol{A}=\sum_{l=0}^{d} \lambda_{l} \boldsymbol{E}_{l}
$$

More generally, taking $p=x^{k}$, each power of $\boldsymbol{A}$ can be expressed as a linear combination of the idempotents $\boldsymbol{E}_{l}$ :

$$
\boldsymbol{A}^{k}=\sum_{l=0}^{d} \lambda_{l}^{k} \boldsymbol{E}_{l}
$$

In [20], Fiol, Garriga and Yebra defined the $i$-local multiplicity of $\lambda_{l} \in$ ev $G$ as the square of the norm of the projection of $\boldsymbol{e}_{i}$ onto $\mathcal{E}_{l}, m_{i}\left(\lambda_{l}\right)=\left\|\boldsymbol{E}_{l} \boldsymbol{e}_{i}\right\|^{2} \geq 0$ (in particular, $\left.m_{i}\left(\lambda_{0}\right)=1 /\|\boldsymbol{v}\|^{2}\right)$. Note that, since $\left\langle\boldsymbol{E}_{l} \boldsymbol{e}_{i}, \boldsymbol{E}_{l} \boldsymbol{e}_{i}\right\rangle=\left\langle\boldsymbol{E}_{l} \boldsymbol{e}_{i}, \boldsymbol{e}_{i}\right\rangle=\left(\boldsymbol{E}_{l}\right)_{i i}$, the $i$-local multiplicity coincides with the $i$-th entry of the diagonal of $\boldsymbol{E}_{l}$. Moreover, if $\mu_{0}=\lambda_{0}>\mu_{1}>\ldots>\mu_{d_{i}}\left(d_{i} \leq d\right)$ are the eigenvalues having nonzero $i$-local multiplicity, the above authors also defined the $i$-local spectrum as:

$$
\operatorname{sp}_{i} G:=\left\{\lambda_{0}^{m_{i}\left(\mu_{0}\right)}, \mu_{1}^{m_{i}\left(\mu_{1}\right)}, \ldots, \mu_{m}^{m_{i}\left(\mu_{d_{i}}\right)}\right\}
$$

The name given to these parameters is justified by the fact that, when the graph "is seen" from a given vertex $i$, its local multiplicities play a role similar to that of the standard multiplicities. The following results from [20] support this claim.

Proposition 3.1 The i-local multiplicities satisfy:

(a) For any vertex $i$,

$$
\sum_{l=0}^{d_{i}} m_{i}\left(\mu_{l}\right)=1
$$

(b) The multiplicity of an eigenvalue of $G$ is the sum, extended to all vertices, of its local multiplicities:

$$
m\left(\lambda_{l}\right)=\sum_{i=1}^{n} m_{i}\left(\lambda_{l}\right) \quad(0 \leq l \leq d) .
$$

Proposition 3.2 Let $i \in V$ be a vertex and let $p$ denote a polynomial. Then,

$$
(p(\boldsymbol{A}))_{i i}=\sum_{l=0}^{d_{i}} m_{i}\left(\mu_{l}\right) p\left(\mu_{l}\right) .
$$

By taking $p=x^{k}$ we obtain the number of circuits of length $k$ through (or rooted at) vertex $i$, namely $\mathcal{C}_{i}(k)=\sum_{l=0}^{d_{i}} m_{i}\left(\mu_{l}\right) \mu_{l}^{k}$.

Other facts support this interpretation of the local spectrum. Given a vertex $i$, its degree satisfies $\delta_{i}=\sum_{l=0}^{d_{i}} m_{i}\left(\mu_{l}\right) \mu_{l}^{2}$; in particular, $C_{i}(2)=\delta_{i}$. Furthermore, analogous to the relation of the diameter and the number of different eigenvalues, the eccentricity of 
a vertex $i$ of a connected graph is bounded above by the number of eigenvalues different from $\lambda_{0}$ in its local spectrum,

$$
\operatorname{ecc}(i) \leq d_{i}=\left|\mathrm{ev}_{i} G\right|-1
$$

Let us define the characteristic polynomial corresponding to the $i$-local spectrum, defined in (24), as

$$
\phi_{i}(x)=\prod_{l=0}^{d_{i}}\left(x-\mu_{l}\right)^{m_{i}\left(\mu_{l}\right)} .
$$

A graph $G$ is said to be spectrally regular if all the vertices have the same local spectrum. The following proposition gives several characterizations of such a graph (see $[11,6])$.

Proposition 3.3 The following statements are equivalent:

(a) $G$ is spectrally regular.

(b) $\phi_{i}=\phi_{j}$ for any $i, j \in V$.

(c) The i-local multiplicities only depend on $\lambda_{l}$, so that, $m_{i}\left(\lambda_{l}\right)=m\left(\lambda_{l}\right) / n$ for any $\lambda_{l} \in \mathrm{ev} G$.

(d) $G$ is walk regular, i.e. $\mathcal{C}_{i}(k)$ only depends on $k$.

(e) $\operatorname{sp} G \backslash i=\operatorname{sp} G \backslash j$ for any $i, j \in V$, where $G \backslash v$ is the graph obtained by deleting the vertex $v$ from $G$.

Moreover, we have

$$
\frac{\phi_{G \backslash i}(x)}{\phi_{G}(x)}=\frac{\phi_{i}^{\prime}(x)}{\phi_{i}(x)}
$$

\subsection{Local distance-regularity and the local predistance polyno- mials}

Let $i \in V$ be a vertex with eccentricity $\operatorname{ecc}(i)=\varepsilon$ of a regular graph $G$. Let $V_{k}:=\Gamma_{k}(i)$ and consider the numbers $c_{k}(j):=\left|\Gamma(j) \cap V_{k-1}\right|, a_{k}(j):=\left|\Gamma(j) \cap V_{k}\right|, b_{k}(j):=\left|\Gamma(j) \cap V_{k+1}\right|$, defined for any $j \in V_{k}$ and $0 \leq k \leq \varepsilon$ (where, by convention, $c_{0}(j)=0$ and $b_{\varepsilon}(j)=0$ for any $\left.j \in V_{\varepsilon}\right)$. We say that $G$ is distance-regular around $i$ whenever $c_{k}(j), a_{k}(j), b_{k}(j)$ do not depend on the considered vertex $j \in V_{k}$ but only on the value of $k$. In such a case, we simply denote them by $c_{k}, a_{k}$ and $b_{k}$, respectively, and we call them the intersection numbers. The matrix

$$
\mathcal{I}(i):=\left(\begin{array}{ccccc}
0 & c_{1} & \cdots & c_{\varepsilon-1} & c_{\varepsilon} \\
a_{0} & a_{1} & \cdots & a_{\varepsilon-1} & a_{\varepsilon} \\
b_{0} & b_{1} & \cdots & b_{\varepsilon-1} & 0
\end{array}\right)
$$

is called the intersection array around vertex $i$. 


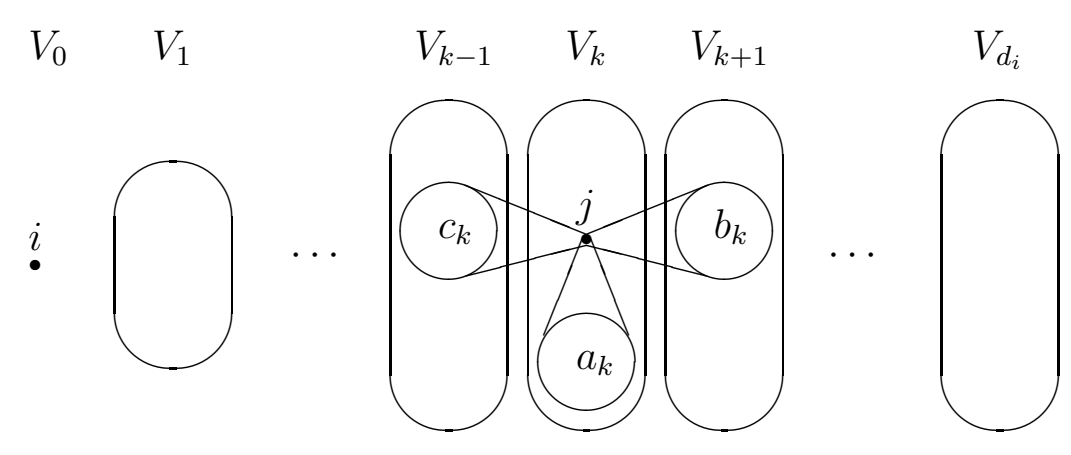

Figure 3: Intersection numbers.

A graph $G$ is distance-regular when it is distance-regular around each of its vertices and with the same intersection array. In other words, if we consider the partition $\Pi(i)$ of $V(G)$ defined by the sets $\Gamma_{k}(i), k=0,1, \ldots, \operatorname{ecc}(i)$, the corresponding quotient $G / \Pi(i)$ is a weighted path with structure independent of the chosen vertex $i$.

Let $G$ be distance-regular around vertex $i \in V$. Note that, for a given vertex $j \in V$, $\left(\boldsymbol{A} \boldsymbol{\rho} V_{k}\right)_{j}=\left|\Gamma(j) \cap V_{k}\right|$. Thus,

$$
\boldsymbol{A} \boldsymbol{\rho} V_{k}=b_{k-1} \boldsymbol{\rho} V_{k-1}+a_{k} \boldsymbol{\rho} V_{k}+c_{k+1} \boldsymbol{\rho} V_{k+1} .
$$

Let $\boldsymbol{A}_{r}, 1 \leq r \leq d$, be the adjacency matrix of $G_{r}$, that is, $\left(\boldsymbol{A}_{r}\right)_{i j}=1$ if $\operatorname{dist}(i, j)=r$ and $\left(\boldsymbol{A}_{r}\right)_{i j}=0$ otherwise. Since $G$ is regular, $\boldsymbol{\rho} V_{r}=\boldsymbol{A}_{r} \boldsymbol{e}_{i}, 1 \leq r \leq d$, and the previous recurrence reads

$$
\boldsymbol{A} \boldsymbol{A}_{k} \boldsymbol{e}_{i}=b_{k-1} \boldsymbol{A}_{k-1} \boldsymbol{e}_{i}+a_{k} \boldsymbol{A}_{k} \boldsymbol{e}_{i}+c_{k+1} \boldsymbol{A}_{k+1} \boldsymbol{e}_{i} .
$$

Thus, the polynomials obtained from the recurrence

$$
x r_{k}=b_{k-1} r_{k-1}+a_{k} r_{k}+c_{k+1} r_{k+1}, \quad \text { with } \quad r_{0}=1, \quad r_{1}=x,
$$

satisfy $r_{k}(\boldsymbol{A}) \boldsymbol{e}_{i}=\boldsymbol{\rho} V_{k}$. Note that, if $G$ is distance-regular, the intersection numbers are the same for all the vertices and we also have $r_{k}(\boldsymbol{A})=\boldsymbol{A}_{k}$, but this is not true in general.

In what follows, we consider the $i$-local scalar product defined as the scalar product associated to the mesh $\mathcal{M}=\operatorname{sp}_{i} G$ and normalized weight function $g_{l}=m_{i}\left(\mu_{l}\right), 0 \leq l \leq d_{i}$ :

$$
\langle f, g\rangle_{i}:=\sum_{l=0}^{d_{i}} m_{i}\left(\mu_{l}\right) f\left(\mu_{l}\right) g\left(\mu_{l}\right)=(f(\boldsymbol{A}) g(\boldsymbol{A}))_{i i},
$$

Lemma 3.4 If $G$ is distance-regular around a vertex $i$, the polynomials $r_{k}, 1 \leq k \leq d$, obtained from the recurrence (25) coincide with the canonical orthogonal system associated to the mesh $\mathcal{M}=\operatorname{sp}_{i} G$ and normalized weight function $g_{l}=m_{i}\left(\mu_{l}\right), 0 \leq l \leq d_{i}$.

Proof. Let us show, first, that the polynomials $r_{k}, 1 \leq k \leq d$ are mutually orthogonal. Notice that the vectors $\rho V_{s}$ and $\rho V_{t}, s \neq t$, are orthogonal. Then, 


$$
\left\langle r_{s}, r_{t}\right\rangle_{i}=\left(r_{s}(\boldsymbol{A}) r_{t}(\boldsymbol{A})\right)_{i i}=\left\langle r_{s}(\boldsymbol{A}) \boldsymbol{e}_{i}, r_{t}(\boldsymbol{A}) \boldsymbol{e}_{i}\right\rangle=\left\langle\boldsymbol{\rho} V_{s}, \boldsymbol{\rho} V_{t}\right\rangle=0 .
$$

Finally, by applying Proposition 2.6, $\operatorname{since} \operatorname{dgr}\left(r_{k}\right)=k$, we conclude the proof.

Given any connected graph $G$ and a vertex of it $i$, in [15] the authors defined the $i$-local predistance polynomials, $p_{k}^{i}, 0 \leq k \leq d_{i}$, as the canonical orthogonal system associated to the mesh $\mathcal{M}=\operatorname{sp}_{i} G$ and normalized weight function $g_{l}=m_{i}\left(\mu_{l}\right), 0 \leq l \leq d_{i}$. As the previous result suggests, these polynomials can be a useful tool to determine whether a graph is distance-regular around a vertex $i$. In this direction, in [20] is proved that $G$ is distance-regular around vertex $i, \operatorname{ecc}(i)=\varepsilon$, if and only if

$$
p_{k}^{i}(\boldsymbol{A}) \boldsymbol{e}_{i}=\boldsymbol{\rho} V_{k}=\sum_{j \in V_{k}} \boldsymbol{e}_{j} \quad(0 \leq k \leq \varepsilon),
$$

generalizing the result in [12] for the so-called predistance polynomials.

Since $m_{i}\left(\lambda_{0}\right)=1 /\|\boldsymbol{v}\|^{2}=1 / n$, we can deduce from (12) that the $i$-local multiplicities of $G$ are given by

$$
m_{i}\left(\mu_{l}\right)=\frac{\pi_{0} p_{d_{i}}^{i}\left(\lambda_{0}\right)}{n \pi_{l} p_{d_{i}}^{i}\left(\mu_{l}\right)} \quad\left(0 \leq l \leq d_{i}\right)
$$

where $\pi_{l}=\prod_{h=0(h \neq l)}^{d_{i}}\left|\mu_{l}-\mu_{h}\right|$, and Corollary 2.7 gives us the value at $\lambda_{0}$ of the highest degree polynomial:

$$
p_{d_{i}}^{i}\left(\lambda_{0}\right)=\left(\sum_{l=0}^{d_{i}} \frac{m_{i}^{2}\left(\lambda_{0}\right) \pi_{0}^{2}}{m_{i}\left(\mu_{l}\right) \pi_{l}^{2}}\right)^{-1} .
$$

Given $i \in V$, we say that a vertex $j \in V$ is $i$-extremal if it is at spectrally maximum distance from it, $\operatorname{dist}(i, j)=d_{i}=\left|\mathrm{ev}_{i} G\right|-1$. The number of $i$-extremal vertices is bounded by

$$
\left|V_{d_{i}}\right| \leq p_{d_{i}}^{i}\left(\lambda_{0}\right)=\left(\sum_{l=0}^{d_{i}} \frac{m_{i}^{2}\left(\lambda_{0}\right) \pi_{0}^{2}}{m_{i}\left(\mu_{l}\right) \pi_{l}^{2}}\right)^{-1},
$$

and, as it is shown in [15], the equality holds if and only if $G$ is distance-regular around vertex $i$.

\subsection{Characterizing distance-regularity}

Let $G$ be a regular graph on $n$ vertices, with spectrum $\operatorname{sp} G=\left\{\lambda=\lambda_{0}, \lambda_{1}^{m_{1}}, \ldots, \lambda_{d}^{m_{d}}\right\}$, $\lambda_{0}>\lambda_{1}>\cdots \lambda_{d}$, and canonical orthogonal system $p_{0}, p_{1}, \ldots, p_{d}$ satisfying Proposition 2.6. In [15], the authors obtained a characterization of distance-regularity through the following results. 
Proposition 3.5 Let $G$ be a graph as above. Let $q_{k}=\sum_{l=0}^{k} p_{l}, 0 \leq k \leq d$ and let $\operatorname{exc}(i)=\left|\Gamma_{d}(i)\right|$ denote the number of vertices at distance $d$ from $i$ (the excess). Then, $G$ is spectrally regular if

$$
\operatorname{exc}(i)=n-q_{d-1}\left(\lambda_{0}\right)=p_{d}\left(\lambda_{0}\right)
$$

for every vertex $i$.

Theorem 3.6 Let $G$ be a spectrally-regular graph. Then the following statements are equivalent:

(a) $G$ is distance-regular around each of its vertices.

(b) $G$ is distance-regular.

As mentioned, in [15], from these two results, jointly with (27), a quasi-spectral characterization of distance-regular graphs is obtained:

Theorem 3.7 (The spectral excess theorem) Let $G$ be a regular graph on $n$ vertices with spectrum $\operatorname{sp} G=\left\{\lambda_{0}, \lambda_{1}^{m_{1}}, \ldots, \lambda_{d}^{m_{d}}\right\}$, and let $p_{0}, p_{1}, \ldots, p_{d}$ be the associated canonical orthogonal system. Then $G$ is distance-regular if and only if, for every vertex $i$,

$$
\operatorname{exc}(i)=p_{d}\left(\lambda_{0}\right)=n\left(\sum_{l=0}^{d} \frac{\pi_{0}^{2}}{m_{l} \pi_{l}^{2}}\right)^{-1}
$$

Using some results from [15, 20], the third author proved in [12] the following result, which gives a bound satisfied by every polynomial, attainable only if the involved graph is distance-regular. It is not unusual that distance-regularity appears when a bound is attained; another example of such a case is the bound given in (27).

Theorem 3.8 Let $G$ be a regular graph with $n$ vertices and $d+1$ distinct eigenvalues. For every vertex $i \in V$, let $s_{d-1}(i)=\left|N_{d-1}(i)\right|=n-\operatorname{exc}(i)$. Then, any polynomial $r \in \mathbb{R}_{d-1}[x]$ satisfies the bound

$$
\frac{r\left(\lambda_{0}\right)^{2}}{\|r\|_{G}^{2}} \leq \frac{n}{\sum_{i \in V} \frac{1}{s_{d-1}(i)}},
$$

and equality is attained if and only if $G$ is a distance-regular graph. Moreover, in this case, we have

$$
\frac{r\left(\lambda_{0}\right)}{\|r\|_{G}^{2}} r=q_{d-1}=\sum_{k=0}^{d-1} p_{k},
$$

where $p_{k}, 0 \leq k \leq d$, are the distance polynomials of $G$.

Note that the above upper bound is, in fact, the harmonic mean of the numbers $s_{d-1}(i)$, $i \in V$. Moreover, in case of equality, $q_{d-1}(\boldsymbol{A})=\sum_{k=0}^{d-1} \boldsymbol{A}_{k}=\boldsymbol{J}-\boldsymbol{A}_{d}$, and the distance- $d$ polynomial of $G$ is just

$$
p_{d}=q_{d}-q_{d-1}=q_{d}-\frac{r\left(\lambda_{0}\right)}{\|r\|_{G}^{2}} r
$$


where $q_{d}$ represents the Hoffman polynomial; that is, $q_{d}=H_{0}=\frac{n}{\pi_{0}} \prod_{l=1}^{d}\left(x-\lambda_{l}\right)$ (see [25]).

Two short proofs of the spectral excess theorem have been recently given by van Dam [8], using the harmonic mean of the numbers $n-\operatorname{exc}(i)$; and the last two authors and Gago [14], by using the mean of the numbers exc $(i)$.

\subsection{Bounding special vertex sets}

Let $i \in V$ be a vertex with eccentricity $\operatorname{ecc}(i)=\varepsilon$. Given integers $k, \mu$ such that $0 \leq k \leq \varepsilon$ and $\mu>0$, let $\Gamma_{k}^{\mu}(i)$ denote the set of vertices which are at distance $k$ from $i \in V$ and from which there exist exactly $\mu$ (shortest) $k$-paths to $i$; so we have the partition $\Gamma_{k}(i)=\cup_{\mu \geq 1} \Gamma_{k}^{\mu}(i)$.

As shown in the following theorem of [10], the $i$-local predistance polynomials can be used to derive some bounds on the cardinality of the set $\Gamma_{k}^{\mu}(i)$, giving also a characterization of the extremal cases. Moreover, when the results are restricted to spectrally regular graphs, we get a proof of a conjecture of Van Dam (see Corollary 3.10 below).

Theorem 3.9 Let $i$ be a vertex of a regular graph $G$, with local spectrum $\mathrm{sp}_{i} G$, and let $\left(p_{k}^{i}\right)_{0 \leq k \leq d_{i}}$ be the $i$-local predistance polynomials. Let $a_{k}$ denote the leading coefficient of $p_{k}^{i}$, and consider the sum polynomials $q_{k}^{i}=\sum_{l=0}^{k} p_{l}^{i}$. For any given integers $\mu>0$ and $0 \leq k<d_{i}$, consider the spectral $k$-excess $e_{k}=n-q_{k}^{i}\left(\lambda_{0}\right)$, and define $\sigma_{k}(\mu):=a_{k} \mu-1$. Then,

$$
\left|\Gamma_{k}^{\mu}(i)\right| \leq \frac{p_{k}^{i}\left(\lambda_{0}\right) e_{k}}{p_{k}^{i}\left(\lambda_{0}\right) \sigma_{k}(\mu)^{2}+a_{k}^{2} \mu^{2} e_{k}}
$$

and equality is attained if and only if either

(a) When $k=\varepsilon$ :

$$
P^{*} \boldsymbol{e}_{i}=\beta_{\varepsilon}^{*} \boldsymbol{j}+\gamma_{\varepsilon}^{*} \boldsymbol{\rho} \Gamma_{\varepsilon}^{\mu}(i)
$$

with the polynomial

$$
P^{*}:=a_{\varepsilon} \mu e_{\varepsilon} p_{\varepsilon}^{i}+p_{\varepsilon}^{i}\left(\lambda_{0}\right) \sigma_{\varepsilon}(\mu) q_{\varepsilon}^{i}
$$

and constants

$$
\beta_{\varepsilon}^{*}:=p_{\varepsilon}^{i}\left(\lambda_{0}\right) \sigma_{\varepsilon}(\mu), \quad \gamma_{\varepsilon}^{*}:=p_{\varepsilon}^{i}\left(\lambda_{0}\right) \sigma_{\varepsilon}(\mu)^{2}+a_{\varepsilon}^{2} \mu^{2} e_{\varepsilon}
$$

(b) When $k<\varepsilon$ :

$$
p_{k}^{i} \boldsymbol{e}_{i}=\boldsymbol{\rho} V_{k},
$$

in which case

$$
n_{k}^{\mu}=n_{k}=p_{k}^{i}\left(\lambda_{0}\right)
$$

In the case of spectrally regular graphs (which implies that $p_{k}^{i}=p_{k}$ for every vertex $i$ ) and $k=d-1$, we have

$$
e_{d-1}=n-q_{d-1}\left(\lambda_{0}\right)=q_{d}\left(\lambda_{0}\right)-q_{d-1}\left(\lambda_{0}\right)=p_{d}\left(\lambda_{0}\right)
$$


and we get

$$
n_{d-1}^{\mu} \leq \frac{p_{d-1}\left(\lambda_{0}\right) p_{d}\left(\lambda_{0}\right)}{p_{d-1}\left(\lambda_{0}\right) \sigma_{d-1}(\mu)^{2}+a_{d-1}^{2} \mu^{2} p_{d}\left(\lambda_{0}\right)} .
$$

When $d=3$ the above proves the following result conjectured by Van Dam in [7].

Corollary 3.10 Let $G$ be a regular graph with four distinct eigenvalues, and predistance polynomials $p_{k}$ with leading coefficients $a_{k}$. Then, for any vertex $i \in V$, the number $n_{2}^{\mu}$ of vertices non-adjacent to $i$, which have $\mu$ common neighbours with $i$, is bounded above by

$$
n_{2}^{\mu} \leq \frac{p_{2}\left(\lambda_{0}\right) p_{3}\left(\lambda_{0}\right)}{p_{2}\left(\lambda_{0}\right)\left(a_{2} \mu-1\right)^{2}+a_{2}^{2} \mu^{2} p_{3}\left(\lambda_{0}\right)} .
$$

Example. Let $G$ be a regular graph with spectrum

$$
\operatorname{sp} G=\left\{4^{1}, 2^{3}, 0^{3},-2^{5}\right\} .
$$

Then $n=12$, and its proper polynomials and their values at $\lambda_{0}=4$ are:

- $p_{0}=1, \quad 1$;

- $p_{1}=x, \quad 4$;

- $p_{2}=\frac{2}{3}\left(x^{2}-x-4\right), \quad \frac{16}{3}$;

- $p_{3}=\frac{1}{12}\left(3 x^{3}-8 x^{2}-16 x+20\right), \quad \frac{5}{3}$;

Then, (37) gives

$$
n_{2}^{\mu} \leq\left\lfloor\frac{20}{7 \mu^{2}-16 \mu+12}\right\rfloor
$$

and hence

$$
\text { - } \mu=0,1,2,3,4, \ldots \Rightarrow n_{2}^{\mu} \leq 1,6,2,0,0, \ldots
$$

An example of a graph with such a spectrum is the one given by Godsil [24] (as an example of walk-regular graph which is neither vertex-transitive nor distance-regular, see Fig. 4) This graph can be constructed as follows: take two copies of the 8-cycle with vertex set $\mathbb{Z}_{8}$ and chords $\{1,5\},\{3,7\}$; joint them by identifying vertices with the same even number and, finally, add edges between vertices labelled with equal odd number. The automorphism group of this graph has two orbits, formed by "even" and "odd" vertices, respectively. For such vertices we have (see again Fig. 4):

- $n_{2}^{\mu}=0,6,1,0,0, \ldots$ (for an even vertex);

- $n_{2}^{\mu}=1,4,2,0,0, \ldots$ (for an odd vertex $)$. 

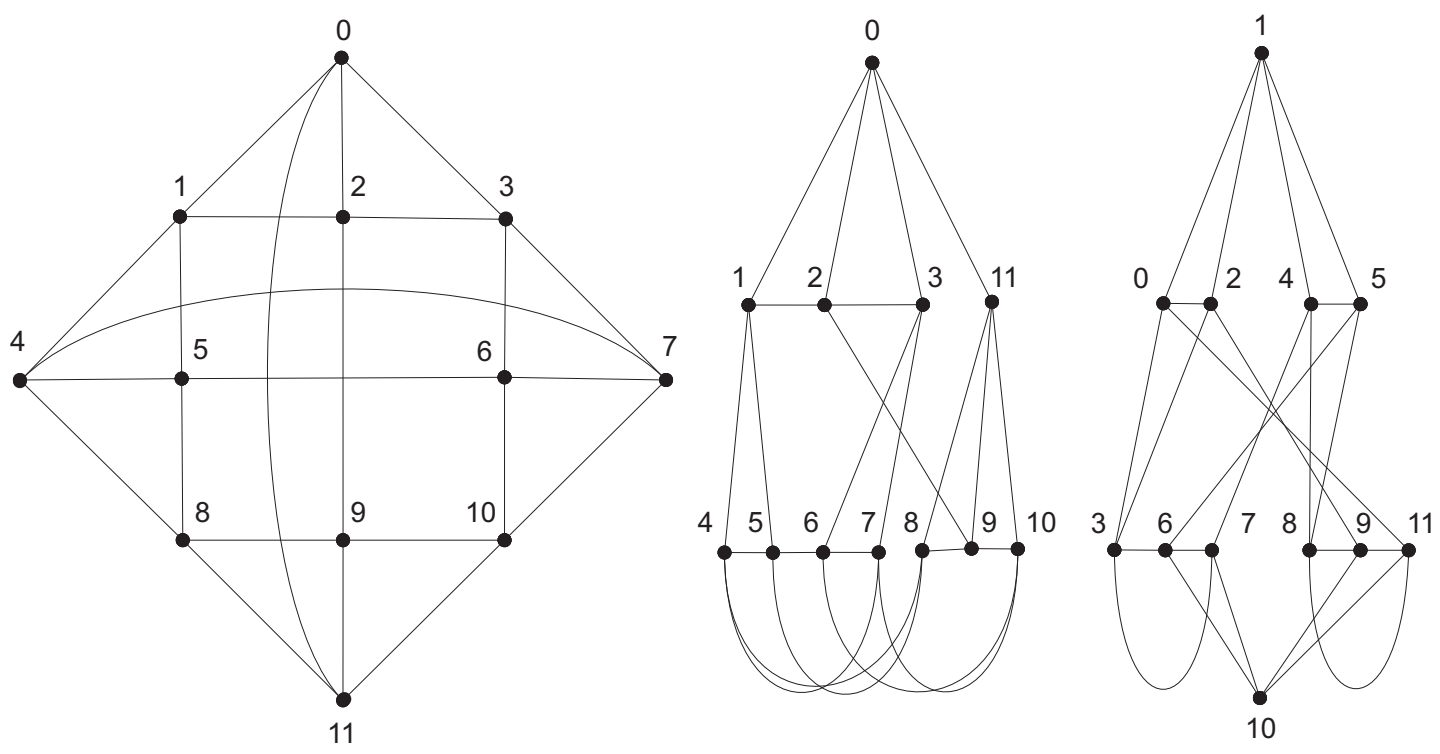

Figure 4: Three drawings of a walk regular graph which is not distance-regular.

\subsection{Representation theory}

Given a graph $G$ and $\lambda_{l} \in \mathrm{ev} G$, we define, for every pair of vertices $i, j$ the $i j$-cosine respect to $\lambda_{l}$ to be

$$
w_{i j}\left(\lambda_{l}\right)=\frac{\left\langle\boldsymbol{E}_{l} \boldsymbol{e}_{i}, \boldsymbol{E}_{l} \boldsymbol{e}_{j}\right\rangle}{\left\|\boldsymbol{E}_{l} \boldsymbol{e}_{i}\right\|\left\|\boldsymbol{E}_{l} \boldsymbol{e}_{j}\right\|}=\frac{m_{i j}\left(\lambda_{l}\right)}{\sqrt{m_{i}\left(\lambda_{l}\right) m_{j}\left(\lambda_{l}\right)}},
$$

where $m_{i j}\left(\lambda_{l}\right)=\left\langle\boldsymbol{E}_{l} \boldsymbol{e}_{i}, \boldsymbol{E}_{l} \boldsymbol{e}_{j}\right\rangle$ is called the crossed (ij-)local multiplicity of $\lambda_{l}$ (see [22]). The name given to this parameter emphasizes the fact that it coincides with the cosine of the angle formed by the projection of the vectors $\boldsymbol{e}_{i}$ and $\boldsymbol{e}_{j}$ onto the eigenspace associated with $\lambda_{l}$. When $G$ is a distance-regular graph, $w_{i j}\left(\lambda_{l}\right)$ only depends on the distance $r=$ $\operatorname{dist}(i, j)$ (see for instance [24]), and it is referred to as the $r$-th cosine $w_{r}\left(\lambda_{l}\right)$. In this case the following recurrence holds

$$
\lambda_{l} w_{r}=c_{r} w_{r-1}+a_{r} w_{r}+b_{r} w_{r+1} \quad(0 \leq r \leq d),
$$

where $c_{r}, a_{r}$, and $b_{r}$ are the intersection parameters of $G ; w_{-1}$ and $w_{d+1}$ are irrelevant (since $c_{0}=b_{d}=0$ ); and $w_{0}=1$.

If $G$ is distance-regular around a vertex $i$ and $\operatorname{dist}(i, j)=r$ the crossed $i j$-local multiplicity of $\mu_{l} \in \operatorname{sp}_{i} G$ can be obtained from the $i$-local predistance polynomials,

$$
m_{i j}\left(\mu_{l}\right)=\frac{p_{r}^{i}\left(\mu_{l}\right)}{p_{r}^{i}\left(\mu_{0}\right)} m_{i}\left(\mu_{l}\right) \quad\left(0 \leq l \leq d_{i}\right)
$$

and hence

$$
w_{i j}\left(\mu_{l}\right)=\frac{p_{r}^{i}\left(\mu_{l}\right)}{p_{r}^{i}\left(\mu_{0}\right)} \sqrt{\frac{m_{i}\left(\mu_{l}\right)}{m_{j}\left(\mu_{l}\right)}} .
$$


So we can infer that in a distance-regular graph the $r$-th cosine is

$$
w_{r}\left(\lambda_{l}\right)=\frac{p_{r}\left(\lambda_{l}\right)}{p_{r}\left(\lambda_{0}\right)} \quad(0 \leq l \leq d)
$$

\subsection{Distance-regularity around a vertex set}

Given a vertex subset $C \subset V$ of a (regular) graph $G$, its normalized vector is defined by

$$
\boldsymbol{e}_{C}:=\frac{1}{\sqrt{|C|}} \rho C=\frac{1}{\sqrt{|C|}} \sum_{i \in C} \boldsymbol{e}_{i}
$$

Consider the spectral decomposition of the vector $\boldsymbol{e}_{C}=\boldsymbol{z}_{0}+\boldsymbol{z}_{1}+\cdots+\boldsymbol{z}_{d}$, where $\boldsymbol{z}_{l} \in$ $\operatorname{Ker}\left(\boldsymbol{A}-\lambda_{l} \boldsymbol{I}\right)$. Similar to the case of the local spectrum, we define the $C$-multiplicity of the eigenvalue $\lambda_{l}$ to be

$$
\begin{aligned}
m_{C}\left(\lambda_{l}\right) & :=\left\|\boldsymbol{z}_{l}\right\|^{2}=\left\|\boldsymbol{E}_{l} \boldsymbol{e}_{C}\right\|^{2}=\left\langle\boldsymbol{E}_{l} \boldsymbol{e}_{C}, \boldsymbol{e}_{C}\right\rangle=\frac{1}{|C|} \sum_{i, j \in C}\left\langle\boldsymbol{E}_{l} \boldsymbol{e}_{i}, \boldsymbol{e}_{j}\right\rangle \\
& =\frac{1}{|C|} \sum_{i, j \in C} m_{i j}\left(\lambda_{l}\right) \quad(0 \leq l \leq d) .
\end{aligned}
$$

The sequence of $C$-multiplicities $\left(m_{C}\left(\lambda_{0}\right), \ldots, m_{C}\left(\lambda_{d}\right)\right)$ corresponds in fact to the socalled "McWilliams transform" of the vector $\boldsymbol{e}_{C}$ (see [24]). Since $\boldsymbol{e}_{C}$ is unitary, we have $\sum_{l=0}^{d} m_{C}\left(\lambda_{l}\right)=1$. If $\mu_{0}\left(=\lambda_{0}\right), \mu_{1}, \ldots, \mu_{d_{C}}$ represent the eigenvalues of $G$ with non-zero $C$-multiplicities, then the (local) $C$-spectrum of $G$ is

$$
\operatorname{sp}_{C} G:=\left\{\lambda_{0}^{m_{C}\left(\lambda_{0}\right)}, \mu_{1}^{m_{C}\left(\mu_{1}\right)}, \ldots, \mu_{d_{C}}^{m_{C}\left(\mu_{d_{C}}\right)}\right\}
$$

where $d_{C}(\leq d)$ is called the dual degree of $C$ (see again [24]).

Notice that we have similar results to those presented in Proposition 3.2 and its direct consequence for the spectrum of a vertex set. For any polynomial $p \in \mathbb{R}[x]$, we have

$$
\begin{aligned}
\left\langle p(\boldsymbol{A}) \boldsymbol{e}_{C}, \boldsymbol{e}_{C}\right\rangle & =\left\langle\sum_{l=0}^{d} p\left(\lambda_{l}\right) \boldsymbol{E}_{l} \boldsymbol{e}_{C}, \boldsymbol{e}_{C}\right\rangle=\sum_{l=0}^{d} p\left(\lambda_{l}\right)\left\langle\boldsymbol{E}_{l} \boldsymbol{e}_{C}, \boldsymbol{e}_{C}\right\rangle \\
& =\sum_{l=0}^{d} m_{C}\left(\lambda_{l}\right) p\left(\lambda_{l}\right)
\end{aligned}
$$

and the number of walks of length $k$ from any vertex of $C$ to any vertex of $C$ is

$$
\mathcal{C}_{C}(k):=\sum_{u, v \in C}\left(\boldsymbol{A}^{k}\right)_{u v}=|C|\left\langle\boldsymbol{A}^{k} \boldsymbol{e}_{C}, \boldsymbol{e}_{C}\right\rangle=|C| \sum_{l=0}^{d} m_{C}\left(\lambda_{l}\right) \lambda_{l}^{k} \quad(k \geq 0)
$$


Proceeding as we did for the local spectrum, the $C$-local predistance polynomials $\left(p_{k}^{C}\right)_{0 \leq k \leq d_{C}}$ are the canonical orthogonal system with respect to the scalar product

$$
\langle f, g\rangle_{C}:=\sum_{l=0}^{d_{C}} m_{C}\left(\mu_{l}\right) f\left(\mu_{l}\right) g\left(\mu_{l}\right)
$$

with normalized weight function $g_{l}=m_{C}\left(\mu_{l}\right)$, satifying $\left\|p_{k}^{C}\right\|_{C}^{2}=p_{k}^{C}\left(\lambda_{0}\right)$. The sum polynomials will be also of our interest, they are defined by $q_{k}^{C}:=\sum_{h=0}^{k} p_{h}^{C}, 0 \leq k \leq d_{C}$. In this context, Theorem 4.2 of [17] reads as follows:

Theorem 3.11 Let $C$ be a vertex subset of a regular graph $G$, with $C$-local predistance polynomials $\left(p_{k}^{C}\right)_{0 \leq k \leq d_{C}}$. Then, for any polynomial $q \in \mathbb{R}_{k}[x]$,

$$
\frac{q\left(\lambda_{0}\right)^{2}}{\|q\|_{C}^{2}} \leq \frac{\left|N_{k}(C)\right|}{|C|}
$$

and equality is attained if and only if

$$
\frac{1}{\|q\|_{C}} q(\boldsymbol{A}) \boldsymbol{e}_{C}=\boldsymbol{e}_{N_{k}}
$$

where $\boldsymbol{e}_{N_{k}}$ represents the unitary characteristic vector of $N_{k}(C)$. Moreover, if this is the case, $q$ is any multiple of $q_{k}^{C}$, whence (39) and (40) become

$$
\begin{aligned}
q_{k}^{C}\left(\lambda_{0}\right) & =\frac{\left|N_{k}(C)\right|}{|C|}, \\
q_{k}^{C}(\boldsymbol{A}) \boldsymbol{\rho} C & =\boldsymbol{\rho} N_{k}(C) .
\end{aligned}
$$

The above result motivated the following definition. A subset $C$ of the vertices of a graph $G$ is tight if $q_{k}^{C}(\boldsymbol{A}) \boldsymbol{\rho} C=\boldsymbol{\rho} N_{k}(C)$ for any $0 \leq k \leq d_{C}$. For every vertex subset $C$, we consider also its antipodal set $\bar{C}$. As the following proposition states, the tight character of a set $C$-or, equivalently, the existence of the $C$-local predistance polynomial $p_{d}^{C}$ leads to the existence of all the $C$-local predistance polynomials with respect to both sets $C$ and $\bar{C}$, and that they are just the members of their associated orthogonal system (see $[17])$.

Proposition 3.12 Let $C$ be a tight set of vertices with predistance orthogonal system $\left(p_{k}\right)_{0 \leq k \leq d_{C}}$, and let $\left(\bar{p}_{k}\right)_{0 \leq k \leq d_{C}}$ be the predistance orthogonal system associated to its antipodal set $\bar{C}$. Then,

(a) The polynomials $\left(\bar{p}_{k}\right)_{0 \leq k \leq d_{C}}$ are just the conjugate polynomials of $\left(p_{k}\right)_{0 \leq k \leq d_{C}}$ :

$$
\bar{p}_{k}=p_{d}^{-1} p_{d-k}, \quad p_{k}=p_{d} \bar{p}_{d-k} \quad\left(0 \leq k \leq d_{C}\right) .
$$

(b) $\bar{C}$ is also tight. (If the family $\mathcal{T}$ of tight vertex sets of a graph $G$ is not empty, then the application which maps every set to its antipodal fixes $\mathcal{T}$ and it is involutive). 


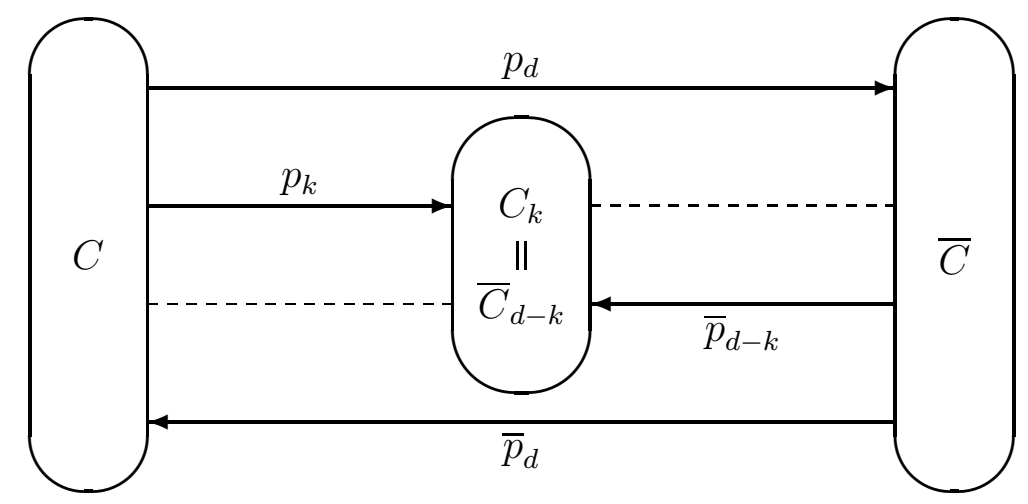

Figure 5: Antipodal tight sets and their distance partition

What is more, for every $0 \leq k \leq d$, the action of the polynomial $p_{k}$ on $\rho C$ coincides with the action of $\bar{p}_{d-k}$ on $\rho \bar{C}$, so revealing the symmetry between the roles of $C$ and $\bar{C}$ (See Fig. 5).

Proposition 3.13 Suppose $C$ is a tight set with antipodal set $\bar{C}$, and $\left(p_{k}\right)_{0 \leq k \leq d},\left(\bar{p}_{k}\right)_{0 \leq k \leq d}$ are the corresponding predistance orthogonal systems. Then,

$$
p_{k} \rho C=\rho C_{k}=\rho \bar{C}_{d-k}=\bar{p}_{d-k} \rho \bar{C} \quad(0 \leq k \leq d) .
$$

\subsection{Completely regular codes in distance-regular graphs}

Let $C \subset V$ be a vertex set. The distance from $C$ to a given vertex $j$ of $V$ is given by $\operatorname{dist}(C, j)=\min _{i \in C}\{\operatorname{dist}(i, j)\}$. This definition gives us a distance partition of $V$ where each part $C_{k}$ is the set of vertices at distance $k$ from $C$. It is natural to say that the eccentricity of $C$ is given by $\operatorname{ecc}(C)=\max _{j \in V}\{\operatorname{dist}(C, j)\}$.

We say that $G$ is distance-regular around $C$, with eccentricity $\varepsilon=\operatorname{ecc}(C)$, if the distance partition $V=C_{0} \cup C_{1} \cup \cdots \cup C_{\varepsilon}$ is regular, that is the numbers

$$
c_{k}(i):=\left|\Gamma(i) \cap C_{k-1}\right|, \quad a_{k}(i):=\left|\Gamma(i) \cap C_{k}\right|, \quad b_{k}(i):=\left|\Gamma(i) \cap C_{k+1}\right|,
$$

where $i \in C_{k}, 0 \leq k \leq \varepsilon$, depend only on the value of $k$, but not on the chosen vertex $i$. The set $C$ is also referred to as a completely regular set or completely regular code (see $[24])$.

There are similar characterizations of distance-regularity around a vertex set to those given for local distance-regularity in previous sections. Analogously to (26), a graph $G$ is distance-regular around a set $C \subset V$, with eccentricity $\varepsilon$, if and only if the $C$-local predistance polynomials $p_{0}^{C}, p_{1}^{C}, \ldots, p_{\varepsilon}^{C}$ satisfy

$$
\boldsymbol{\rho} C_{k}=p_{k}^{C}(\boldsymbol{A}) \boldsymbol{\rho} C \quad(0 \leq k \leq \varepsilon) .
$$

In [18] the authors gave the following characterization of completely regular codes in terms of the number of vertices at spectrally maximum distance from $C$. From this point of view it can be seen as the analogue of Theorem 3.7. 
Theorem 3.14 Let $G=(V, E)$ be a regular graph. A vertex subset $C \subset V$, with $r$ vertices and local spectrum $\operatorname{sp}_{C} G=\left\{\lambda_{0}^{m_{C}\left(\lambda_{0}\right)}, \mu_{1}^{m_{C}\left(\mu_{1}\right)}, \ldots, \mu_{d_{C}}^{m_{C}\left(\mu_{d_{C}}\right)}\right\}$, is a completely regular code if and only if the number of vertices at distance $d_{C}$ from $C$; that is, $n_{d_{C}}(C):=\left|C_{d_{C}}\right|$ satisfies

$$
\begin{aligned}
n_{d_{C}}(C)=p_{d_{C}}^{C}\left(\lambda_{0}\right) & =r\left(\sum_{l=0}^{d_{C}} \frac{m_{C}\left(\lambda_{0}\right)^{2} \pi_{0}^{2}}{m_{C}\left(\mu_{l}\right) \pi_{l}^{2}}\right)^{-1} \\
& =\frac{n^{2}}{r}\left(\sum_{l=0}^{d_{C}} \frac{\pi_{0}^{2}}{m_{C}\left(\mu_{l}\right) \pi_{l}^{2}}\right)^{-1} .
\end{aligned}
$$

The number of ordered pairs $(i, j)$ of vertices from $C$ which are $k$ apart in a distanceregular graph $G$ is given by

$$
\sum_{i, j \in C}\left(\boldsymbol{A}_{k}\right)_{i j}=\left\langle\boldsymbol{A}_{k} \boldsymbol{e}_{C}, \boldsymbol{e}_{C}\right\rangle=|C|\left\langle p_{k}(\boldsymbol{A}) \boldsymbol{e}_{C}, \boldsymbol{e}_{C}\right\rangle=|C| \sum_{l=0}^{d} m_{C}\left(\lambda_{l}\right) p_{k}\left(\lambda_{l}\right)
$$

From this we see that, within $C$, the mean number of vertices at distance $k$ (from a vertex) in $G$ is

$$
\bar{n}_{k}:=\frac{1}{|C|} \sum_{i \in C}\left|\Gamma_{k}(i) \cap C\right|=\sum_{l=0}^{d} m_{C}\left(\lambda_{l}\right) p_{k}\left(\lambda_{l}\right) \quad(0 \leq k \leq d) .
$$

The numbers $\bar{n}_{k}, 0 \leq k \leq d$, are called the inner distribution of $C$ and, as commented by Godsil [24], they represent the probability, through the ratio $\bar{n}_{k} /|C|$, that a randomly chosen pair of vertices from $C$ are at distance $k$. Notice that always $\bar{n}_{0}=1$ and $\sum_{k=0}^{d} \bar{n}_{k}=$ $|C|$.

These last two observations lead us to conclude that, in a distance regular graph, the local multiplicities of a vertex subset $C$ can be easily computed from the distance polynomials of $G$, its spectrum, and the inner distribution of $C$ (see [18]).

Proposition 3.15 Let $G$ be a distance-regular graph with a given subset $C$ of $r$ vertices. Then there exist nonnegative numbers $r_{0}(=1), r_{1}, \ldots, r_{k}$, such that $\bar{n}_{k}=r_{k}$ for every $0 \leq k \leq d$, if and only if the $C$-multiplicities satisfy

$$
m_{C}\left(\lambda_{l}\right)=\frac{m\left(\lambda_{l}\right)}{n} \sum_{k=0}^{d} r_{k} \frac{p_{k}\left(\lambda_{l}\right)}{p_{k}\left(\lambda_{0}\right)} \quad(0 \leq l \leq d)
$$

In particular $m_{C}\left(\lambda_{0}\right)=\frac{r}{n}$ and if $C$ is a single vertex, $C=\{i\}$, we have $r_{0}=1, r_{1}=\cdots=$ $r_{d}=0$, so $m_{i}\left(\lambda_{l}\right)=\frac{m\left(\lambda_{l}\right)}{n} \frac{p_{0}\left(\lambda_{l}\right)}{p_{0}\left(\lambda_{0}\right)}=\frac{m\left(\lambda_{l}\right)}{n}$, as expected.

In the case when all vertices in $C$ are at distance $k$ from each other, (46) gives:

$$
m_{C}\left(\lambda_{l}\right)=\frac{m\left(\lambda_{l}\right)}{n}\left(1+(r-1) \frac{p_{k}\left(\lambda_{l}\right)}{p_{k}\left(\lambda_{0}\right)}\right) \quad(0 \leq l \leq d) .
$$


But, when $k=d$, we know from Corollary 2.7 that $p_{d}\left(\lambda_{l}\right)<0$ for every odd $l$. This fact, together with $m_{C}\left(\lambda_{l}\right) \geq 0$, yields the following upper bound for the maximum number of vertices mutually at maximum distance $d$ :

$$
r \leq 1+\min _{l=1,3, \ldots} \frac{p_{d}\left(\lambda_{0}\right)}{\left|p_{d}\left(\lambda_{l}\right)\right|}
$$

A similar upper bound is given in [6] for the more general case of spectrally regular graphs.

\section{References}

[1] E. Bannai and T. Ito, Algebraic Combinatorics I: Association Schemes. Benjamin/Cummings, Menlo Park, CA, 1984.

[2] N. Biggs, Algebraic Graph Theory. Cambridge University Press, Cambridge, 1974; second edition: 1993.

[3] A.E. Brouwer, A.M. Cohen and A. Neumaier, Distance-Regular Graphs. SpringerVerlag, Berlin, 1989.

[4] T.S. Chihara, An Introduction to Orthogonal Polynomials, Gordon and Breach, New York, 1978.

[5] F.R.K. Chung, V. Faber, and T.A. Manteuffel, An upper bound on the diameter of a graph from eigenvalues associated with its Laplacian, SIAM J. Discrete Math. 7 (1994), 443-457.

[6] C. Dalfó, M.A. Fiol and E. Garriga, On k-walk-regular graphs, European J. Combin., submitted.

[7] E. R. van Dam, Graphs with few eigenvalues, Ph.D. Thesis, Tilburg University, 1996.

[8] E. R. van Dam, The spectral excess theorem for distance-regular graphs: a global (over)view, Electron. J. Combin. 15 (2008), no. 1, Research Paper 129, 10 pp. (electronic).

[9] E.R. van Dam and W.H. Haemers, Eigenvalues and the diameter of graphs, Linear Multilinear Algebra 39 (1995), 33-44.

[10] M.A. Fiol, Some applications of the proper and adjacency polynomials in the theory of graph spectra, Electron. J. Combin. 4 (1997), no. 1, Research Paper 21, 24 pp. (electronic).

[11] M.A. Fiol, On pseudo-distance-regularity, Linear Algebra Appl. 323 (2001), 145-165.

[12] M.A. Fiol, Algebraic characterizations of distance-regular graphs, Discrete Math. 246 (2002), 111-129.

[13] M.A. Fiol, Spectral bounds and distance-regularity, Linear Algebra Appl. 397 (2005), $17-33$.

[14] M.A. Fiol, S. Gago and E. Garriga, A new approach to the spectral excess theorem for distance-regular graphs, Linear Algebra Appl., accepted (2009). 
[15] M.A. Fiol and E. Garriga, From local adjacency polynomials to locally pseudodistance-regular graphs, J. Combin. Theory Ser. B 71 (1997), no. 2, 162-183.

[16] M.A. Fiol and E. Garriga, The alternating and adjacency polynomials, and their relation with the spectra and diameters of graphs, Discrete Appl. Math. 87 (1998), no. $1-3,77-97$.

[17] M.A. Fiol and E. Garriga, On the algebraic theory of pseudo-distance-regularitiy around a set, Linear Algebra and its Applications 298 (1999), 115-141.

[18] M.A. Fiol and E. Garriga, An algebraic characterization of completely regular codes in distance-regular graphs, SIAM J. Discrete Math. 15 (2002), no. 1, 1-13.

[19] M.A. Fiol, E. Garriga, and J.L.A. Yebra, On a class of polynomials and its relation with the spectra and diameters of graphs, J. Combin. Theory Ser. B 67 (1996), $48-61$.

[20] M.A. Fiol, E. Garriga, and J.L.A. Yebra, Locally pseudo-distance-regular graphs, J. Combin. Theory Ser. B 68 (1996), 179-205.

[21] M.A. Fiol, E. Garriga and J.L.A. Yebra, From regular boundary graphs to antipodal distance-regular graphs, J. Graph Theory 27 (1998), no. 3, 123-140.

[22] M.A. Fiol, E. Garriga, and J.L.A. Yebra, Boundary graphs: The limit case of a spectral property, Discrete Math. 226 (2001), no. 1-3, 155-173.

[23] E. Garriga, Contribució a la Teoria Espectral de Grafs: Problemes Mètrics i Distancia-Regularitat. (Catalan) Ph.D. Thesis, Universitat Politècnica de Catalunya (1997).

[24] C.D. Godsil, Algebraic Combinatorics. Chapman and Hall, New York, 1993.

[25] A.J. Hoffman, On the polynomial of a graph, Amer. Math. Monthly 70 (1963), 30-36.

[26] P. Rowlinson, Linear algebra, in: Graph Connections (ed. L.W. Beineke and R.J. Wilson), Oxford Lecture Ser. Math. Appl., Vol. 5, 86-99, Oxford Univ. Press, New York, 1997.

[27] G. Szegö, Orthogonal Polynomials, Fourth edition, Amer. Math. Soc., Providence, R.I., 1975. 